\title{
SINGULAR REDUCTION OF RESONANT HAMILTONIANS
}

\author{
KENNETH R. MEYER, JESÚS F. PALACIÁN, AND PATRICIA YANGUAS
}

\begin{abstract}
We investigate the dynamics of resonant Hamiltonians with $n$ degrees of freedom to which we attach a small perturbation. Our study is based on the geometric interpretation of singular reduction theory. The flow of the Hamiltonian vector field is reconstructed from the cross sections corresponding to an approximation of this vector field in an energy surface. This approximate system is also built using normal forms and applying reduction theory obtaining the reduced Hamiltonian that is defined on the orbit space. Generically, the reduction is of singular character and we classify the singularities in the orbit space, getting three different types of singular points. A critical point of the reduced Hamiltonian corresponds to a family of periodic solutions in the full system whose characteristic multipliers are approximated accordingly to the nature of the critical point.
\end{abstract}

\section{INTRODUCTION}

We present a way of studying perturbed resonant Hamiltonian systems with $n$ degrees of freedom $(n \geq 2)$ that we have developed during the last few years. It is a very classical problem that has been approached from different points of view by many authors, see for instance $[55,51,12,33,34,16,56,2]$ or [23] and references therein. As in our previous works the point of view is qualitative, and we provide results on the existence of periodic solutions, their stability and possible bifurcations. The procedure can be applied to study the stability of equilibria or the existence of invariant tori. We have tried to present a methodology to analyze resonant Hamiltonians which exposes our results in an intuitive manner.

The features of the software MATHEMATICA have been applied to perform all the computations and make the pictures that are essential in the understanding of the problem.

In [45] we give some preliminary results on the existence of families of periodic solutions and their bifurcations for two degrees of freedom Hamiltonians in semi-simple resonance $k:-1$ ( $k$ a positive integer). These solutions are typically found in the planar circular restricted three body problem around the Lagrange equilateral equilibria $\mathcal{L}_{4}$ and $\mathcal{L}_{5}$.

Our primary goal is to look at classical perturbation theory through the lens of invariant theory and singular reduction introduced in [3], with the goal of exposing some of the underlying geometry. The papers $[57,35,2]$ and the book [8] are also relevant in the context of singular reduction theory. Previous approaches using this perspective can be found in the literature; see, for instance [6]. The case of regular reduction was addressed in [44,61]. Here we study small perturbations of maximally super-integrable systems and our prime example is perturbations of a system of two harmonic oscillators with rationally related frequencies. Examples of Hamiltonians with more than two degrees of freedom will be given in [46].

Let $\mathcal{H}: \mathcal{M} \rightarrow \mathbb{R}$ be a smooth Hamiltonian of $n$ degrees of freedom on a symplectic manifold $\mathcal{M}$ of dimension $2 n$. Since $\mathcal{H}$ is an integral the set $\mathcal{N}=\mathcal{N}_{h}=\{z \in \mathcal{M}: \mathcal{H}(z)=h\}$ is a smooth invariant submanifold of dimension $2 n-1$ when $h \in \mathbb{R}$ is a regular value. The orbit space $\mathbb{O}=\mathbb{O}_{h}$ is the quotient space obtained from $\mathcal{N}$ by identifying orbits to a point. In general quotient spaces are not even Hausdorff, but in some important examples in mechanics the orbit space is a manifold of dimension $2 n-2$. For example Moser [47] showed that the orbit space for the regularized spatial Kepler problem with negative energy is $S^{2} \times S^{2}$. What is essential for Moser Theorem and our example treated below is that all the solutions are periodic so that $\mathcal{N}$ is foliated by circles. However, in our case not all the solutions have the same period and

Date: May 9, 2018.

1991 Mathematics Subject Classification. 34C20, 34C25, 37J40, 70F10, 70K65.

Key words and phrases. normal form and resonant Hamiltonian; singular reduction, cross section, orbit space and orbifold; polynomial invariants and Poisson algebra; plateau, peak and ridge; symplectic coordinates and symplectic smoothing; periodic solution, characteristic multipliers, bifurcation. 
so our orbit space is a symplectic orbifold. Another example of a system whose solutions are all periodic is the case of $n$ harmonic oscillators with the same frequency. Moser [47] handled this problem, proving that the orbit space is the complex projective space $\mathbb{C P}^{n-1}$.

We attack our problem from two points of view. First, the periodic solutions of $\mathcal{H}$ define a symplectic Lie group action $\mathcal{Z}: \mathcal{S} \times \mathcal{M} \rightarrow \mathcal{M}$ where $\mathcal{S}$ is just the circle group so the action is proper and locally free. With this we show that the orbit space $\mathbb{O}=\mathcal{N} / \mathcal{S}$ is a symplectic orbifold by viewing cross sections to the flow as symplectic charts on $\mathbb{O}$. Related recent work is presented by Dullin et al. [13]. These authors focus on diffeomorphisms and introduce the concept of reduction by lifting. They show that after finding a global Poincaré section of the symmetry flow, there are coordinates in which the map takes a reduced form allowing for a decrease of dimensionality. A Hamiltonian function that is invariant under the action $\mathcal{Z}$ is well defined on $\mathbb{O}$ and is called reduced Hamiltonian, while its associated equations of motion are called reduced system. In many cases a normal form computation together with a truncation of the tail is carried out in order to get a Hamiltonian with a continuous symmetry so that reduction can be applied [61].

Second, we study the integrals and the invariant spaces defined by them. Any collection of harmonic oscillators is integrable if the system has $n$ independent integrals in involution but, due to resonance, our problem is super-integrable, i.e. it has more than $n$ independent integrals $[37,19,20]$. Actually our system has $2 n-1$ independent integrals, thus it is called maximally super-integrable [25, 26]. In this latter case one has to study the reduced Hamiltonian system with $n-1$ degrees of freedom obtained after averaging a given perturbation along the periodic solutions of the unperturbed system [53,61], then reducing by the acquired $S^{1}$-symmetry. As the perturbation analysis has to be performed in a space of dimension $2 n-2$ it becomes more complicated. We proceed by means of the polynomial invariants associated to the reduction. In the particular case of resonant Hamiltonians this leads to get $3 n-2$ polynomial invariants with $n-1$ constraints and a model for $\mathbb{O}$ as a semi-algebraic variety embedded in $\mathbb{R}^{3 n-2}$. Besides the $n-1$ constraints one has to add some inequalities that the invariants are required to fulfill so that $\mathbb{O}$ makes sense, as we shall show with more detail in the forthcoming sections. The dimension of the orbit space is always $2 n-2$.

When $n=2$ we shall provide local symplectic coordinates for both the regular and singular points of (O) These coordinates are related to the polynomial invariants and are obtained from the action-angle coordinates. They are useful in the analysis of the dynamics of the reduced system, for instance we use them to establish the stability and bifurcations of the critical points of the Hamiltonian. An interesting feature of the coordinates that we introduce is that they desingularize the singular points of the orbifold $\mathbb{O}$. The corresponding symplectic coordinates for the general case $n \geq 2$ will be given in [46].

Another goal of our study is to present a classification of the types of points occurring in the orbit spaces resulting from the reduction of resonant Hamiltonians with $n$ degrees of freedom. Concretely we distinguish among plateau, peaks and ridges. The plateau is the set of regular points of the orbifold, the peaks are isolated singular points and the ridges correspond to the singular points lying in sets of dimensions greater than or equal to 1 . In a peak only one of the $n$ principal modes of the unperturbed Hamiltonian is non-zero and it is always a critical point of the reduced Hamiltonian. A ridge may or may not be composed of critical points of the reduced system and at least two modes are non-zero and interact. The main purpose of our classification is that in the reconstruction of the periodic solutions related to the critical points of the orbit space, the characteristic multipliers of these solutions are approximated differently, depending on whether they correspond to plateau, peaks or ridges. An extra singularity of $\mathbb{O}$ happens in the zero level set of the unperturbed Hamiltonian when it corresponds to an indefinite quadratic form in rectangular coordinates. This singularity corresponds to the origin in $\mathbb{R}^{2 n}$.

We provide two examples for $n=2$ in detail which illustrate that our approach is particularly useful when there are many parameters and bifurcations. The flows come from normalized Hamiltonians which are truncated at a convenient order. They are depicted for different values of the parameters by using MATHEMATica to exhibit different parametric bifurcations occurring in each example. In addition to that, these approximate flows can be understood as cross sections to the flow of the full problem in adequate symplectic charts on the orbit space.

In our approach we survey some known theory combining it with new results. In this respect the main achievements of our development are: (i) We provide a new insight of regular and singular reduction of resonant Hamiltonians from the point of view of cross sections and how this procedure is related to the 
reduction theory of symmetric Hamiltonians. (ii) When $n=2$ we give a methodology to obtain symplectic coordinates in the orbit space around critical points that are either regular or singular, considering all the possible cases. In the singular case the new coordinates desingularize the orbit space in a neighborhood of the critical point. (iii) We classify the possible singularities of resonant Hamiltonians with $n$ degrees of freedom, providing new results about the characteristic multipliers of the periodic solutions related to these singularities.

The case $n>2$ deserves a deeper insight and we shall give more theoretical results as well as related examples in [46]. In particular the general case requires to handle techniques from computer algebra theory in order to achieve the analysis of resonances successfully.

The paper is structured in seven sections. In Section 2 we deal with the unperturbed fully resonant Hamiltonian with $n$ degrees of freedom in rectangular and action-angle coordinates. Section 3 is devoted to the study of the orbit space through cross sections for arbitrary $n$, but specializing later on for $n=2$. For the maximally super-integrable resonant Hamiltonian, $\mathcal{H}_{R}$, we obtain the set of polynomial invariants from the independent integrals and then the orbit space defined by these invariants in Section 4. Also the classification of the different types of points in the orbit space $\mathbb{O}$ is given. Moreover the case $n=2$ is studied in full detail, analyzing all possible cases of the orbifolds, which in this circumstance are 2-dimensional surfaces. In Section 5 we introduce symplectic coordinates for the $q: p$ resonance, dealing with both regular and singular points. The case of small perturbations attached to a Hamiltonian with $n$ degrees of freedom is studied in Section 6 where the type of the characteristic multipliers of the periodic solutions is determined from the critical

points in $\mathbb{O}$. In addition to that, the case of the extremal bifurcation (also called saddle-center bifurcation) of periodic solutions is tackled from the point of view of reduction theory. The purpose of Section 7 is to study the existence and bifurcations of periodic solutions for Hamiltonian systems in resonances 2:-1 and $3: 2$.

\section{The Unperturbed System}

Consider a quadratic Hamiltonian which gives rise to a linear Hamiltonian system of differential equations, specifically let $\mathcal{H}_{R}=\frac{1}{2} z^{T} S z$ and $\dot{z}=A z$ where $z \in \mathbb{R}^{2 n}, S$ is a $2 n \times 2 n$ symmetric matrix, $A=\mathcal{J} S$ a Hamiltonian matrix and $\mathcal{J}$ is the standard $2 n \times 2 n$ skew symmetric matrix

$$
\mathcal{J}=\left[\begin{array}{rr}
0 & I \\
-I & 0
\end{array}\right]
$$

Here $\mathcal{N}=\left\{z \in \mathbb{R}^{2 n}: \mathcal{H}_{R}(z)=h, z \neq 0\right\}$.

Let the Hamiltonian matrix $A$ be semi-simple and have only pure imaginary eigenvalues that are all rational multiples of one another. More specifically, let the eigenvalues be

$$
\pm k_{1} \omega \mathrm{i}, \pm k_{2} \omega \mathrm{i}, \ldots, \pm k_{n} \omega \mathrm{i}
$$

where $\omega$ is positive real, $k_{i} \in \mathbb{Z} \backslash\{0\}$, with $\operatorname{gcd}\left(k_{1}, k_{2}, \ldots, k_{n}\right)=1$. By a change of the time scale we may take $\omega=1$. In this case the Hamiltonian can be put into the form

$$
\mathcal{H}_{R}(x, y)=\frac{1}{2}\left[k_{1}\left(x_{1}^{2}+y_{1}^{2}\right)+k_{2}\left(x_{2}^{2}+y_{2}^{2}\right)+\cdots+k_{n}\left(x_{n}^{2}+y_{n}^{2}\right)\right],
$$

where $z=(x, y) \in \mathbb{R}^{n} \times \mathbb{R}^{n}$.

Let $Z(t)=e^{A t}$ be the fundamental matrix solution all of whose entries are $\cos k_{j} t$ or $\sin k_{j} t$ so, they have period $2 \pi /\left|k_{j}\right|$. Since the gcd of the $k_{i}$ 's is 1 , the matrix $Z(t)$ is periodic of period $2 \pi$. Thus $\mathcal{N}$ is foliated by circles. Let $\mathcal{S}=\mathbb{R}^{1} /(2 \pi)$ so that $Z$ defines a symplectic Lie group action

$$
\mathcal{Z}: \mathcal{S} \times \mathcal{N} \rightarrow \mathcal{N}:(t, z) \mapsto Z(t) z
$$

Since $\mathcal{S}$ is just the circle group it is compact and the action is proper. Let $\mathbb{O}=\mathcal{N} / \mathcal{S}$ be the orbit space and $\Pi: \mathcal{N} \rightarrow \mathbb{O}$ the projection map.

Change to action-angle coordinates by $I_{j}=x_{j}^{2}+y_{j}^{2}, \theta_{j}=\tan ^{-1}\left(y_{j} / x_{j}\right)$ which is symplectic with multiplier 2 so that the Hamiltonian becomes

$$
\mathcal{H}_{R}(I, \theta)=k_{1} I_{1}+k_{2} I_{2}+\cdots+k_{n} I_{n}
$$


and in these coordinates the equations of motion are

$$
\dot{I}_{j}=0, \quad \dot{\theta}_{j}=-k_{j} .
$$

(This is a slight variation of the usual definition of action-angle variables that reduces the number of 2's in the paper.)

\section{Orbit Space by Cross Sections}

3.1. Orbifolds, Sections and Poincaré Maps. In general the orbit space of a proper locally free Lie group action on a manifold is an orbifold, see [54] where the concept of orbifold was introduced with the name $V$-manifold. It is easy to check the conditions for $\mathbb{O}$ to be an orbifold and in fact it can be inferred from our discussion. If the manifold and the action are both symplectic then so is the orbit space. See [36] for all the definitions related to symplectic orbifolds.

The basic tool used to prove these results depends on the concept of a slice, a group action invariant neighborhood of an orbit. However, since we are interested in bifurcation theory our key concept is a cross section in an energy surface which we use here. We will simply say section for cross section in an energy surface. It is well known that sections are symplectic, the flow induced map between sections, the Poincaré map, is symplectic and fixed points in the section maps often correspond to periodic solutions [43].

Now we will show that $\mathbb{O}$ is a symplectic orbifold by giving an atlas of symplectic charts. Use action-angle coordinates and let $d \in \mathbb{O}$ and $\left(I_{1}^{*}, \ldots, I_{n}^{*}, \theta_{1}^{*}, \ldots, \theta_{n}^{*}\right) \in \Pi^{-1}(d)$. At least one of the $I_{i}^{*}$ 's is nonzero so for simplicity let $I_{1}^{*} \neq 0$ and $k_{1}>0$, and let $T=2 \pi / k_{1}$. Take $\theta_{1}=0$ as a section in the level $\mathcal{H}_{R}=h$ and let this section be $F \subset \mathcal{N}$ with $F$ restricted by the inequalities

$$
\frac{1}{2} \delta<\left|I_{i}-I_{i}^{*}\right|<\delta \quad \text { if } \quad I_{i}^{*} \neq 0 \quad \text { or } \quad 0 \leq\left|I_{i}-I_{i}^{*}\right|<\delta \quad \text { if } \quad I_{i}^{*}=0
$$

with $\delta$ small. Also let $P^{\prime}: F \rightarrow F$ be the Poincaré map. Let $U \subset \mathbb{R}^{2 n-2}$ be the open set with action-angle variables $\left(I_{2}, \ldots, I_{n}, \theta_{2}, \ldots, \theta_{n}\right)$ with the same restriction on the $I_{i}$ 's as above and let

$$
\Psi: U \rightarrow F:\left(I_{2}, \ldots, I_{n}, \theta_{2}, \ldots, \theta_{n}\right) \mapsto\left(I_{1}, I_{2}, \ldots, I_{n}, 0, \theta_{2}, \ldots, \theta_{n}\right)
$$

be the prechart with $I_{1}$ determined by $\mathcal{H}_{R}=h$.

The section map $P=\Psi^{-1} \circ P^{\prime} \circ \Psi$ as a mapping of $U$ is

$$
\begin{aligned}
& I_{2} \mapsto I_{2}, \ldots, I_{n} \mapsto I_{n} \\
& \theta_{2} \mapsto \theta_{2}-\left(k_{2} / k_{1}\right) 2 \pi, \ldots, \theta_{n} \mapsto \theta_{n}-\left(k_{n} / k_{1}\right) 2 \pi
\end{aligned}
$$

which is a linear symplectic map written in action-angle coordinates.

Clearly $P^{k_{1}}=$ id, i.e. the identity map, and let $P^{\kappa}=$ id where $\kappa$ is a minimal divisor of $k_{1}$ with that property. If $a \in U$ then the orbit through $a$ meets the set $U$ in the points $a, P(a), P^{2}(a), \ldots, P^{\kappa-1}(a)$, so in the orbit space these points are identified to one point. Let $G=\left\{P^{0}=\right.$ id, $\left.P, P^{2}, \ldots, P^{\kappa-1}\right\}$ be the group of linear symplectic maps of $U$ and define $\tilde{\Psi}: U / G \rightarrow \mathbb{O}$. The map $\tilde{\Psi}$ is a homeomorphism and it is a symplectic orbifold chart at $d$.

The identification defined by $\tilde{\Psi}$ pinches the cross section forming what we will call a peak or a ridge. This is illustrated later in this section for $n=2$ in Figures 2 and 4. In the later section on symplectic smoothing, Section 5.2, we show how to reconstruct the cross section so that we can apply classical perturbation theory.

Since the Poincaré map from one section to another is symplectic then given two such precharts $\Psi_{i}: U_{i} \rightarrow$ $\mathbb{O}, i=1,2$ the mapping $\Psi_{1}^{-1} \circ \Psi_{2}$ is symplectic where defined. The collection of such orbifold charts is a symplectic atlas.

The non-trivial multipliers of the periodic solution corresponding to $d \in \mathbb{O}$ are

$$
e^{ \pm\left(k_{2} / k_{1}\right) 2 \pi \mathrm{i}}, \ldots, e^{ \pm\left(k_{n} / k_{1}\right) 2 \pi \mathrm{i}}
$$

see more details on characteristic multipliers in $[43,61]$. We shall return to this issue in Section 6 where it will be shown how the multipliers are affected when a small perturbation is attached to the Hamiltonian $\mathcal{H}_{R}$. 
3.2. Models for $q: p$ Resonance. Look at the two degrees of freedom example by setting $k_{1}=q, k_{2}=p$ with $\operatorname{gcd}(q, p)=1$ and $q>0, p \neq 0$. The resonant harmonic oscillator is expressed by the Hamiltonian

$$
\mathcal{H}_{q p}=\frac{1}{2}\left[q\left(x_{1}^{2}+y_{1}^{2}\right)+p\left(x_{2}^{2}+y_{2}^{2}\right)\right]=q I_{1}+p I_{2},
$$

where $x_{1}, x_{2}, y_{1}, y_{2}$ are rectangular coordinates and $I_{1}, I_{2}, \theta_{1}, \theta_{2}$ are action-angle coordinates. If $q<0$ we change the sign of the Hamiltonian.

Model for $\mathcal{N}$ When $q>0, p>0$. We can use action-angle variables to introduce coordinates on the sphere $\mathcal{N}$, provided we are careful to observe the conventions of polar coordinates: (i) $I_{1} \geq 0, I_{2} \geq 0$; (ii) $\theta_{1}$ and $\theta_{2}$ are defined modulo $2 \pi$; and (iii) $I_{1}=0$ or $I_{2}=0$ corresponds to a point. For a careful explanation of these conventions see [43], Sections 1.8 and 1.9.

For simplicity let $h=1$. Starting with the symplectic coordinates $I_{1}, \theta_{1}, I_{2}, \theta_{2}$ for $\mathbb{R}^{4}$, we note that since $\mathcal{H}_{q p}=q I_{1}+p I_{2}=h=1$ on $\mathcal{N}$ we can discard $I_{2}$ and make the restriction $0 \leq I_{1} \leq 1 / q$ (note that $I_{2}$ will return). We use $I_{1}, \theta_{1}, \theta_{2}$ as coordinates on $\mathcal{N}$. Now $I_{1}, \theta_{1}$ with $0 \leq I_{1} \leq 1 / q$ are just coordinates for the closed unit disk in $\mathbb{R}^{2}$ which is drawn in green in Figure $1(\mathrm{~b})$. For each point of the open disk, there is a circle with coordinate $\theta_{2}($ defined $\bmod 2 \pi)$, but $I_{2}=0$ when $I_{1}=1 / q$, so the circle collapses to a point over the boundary of the disk.

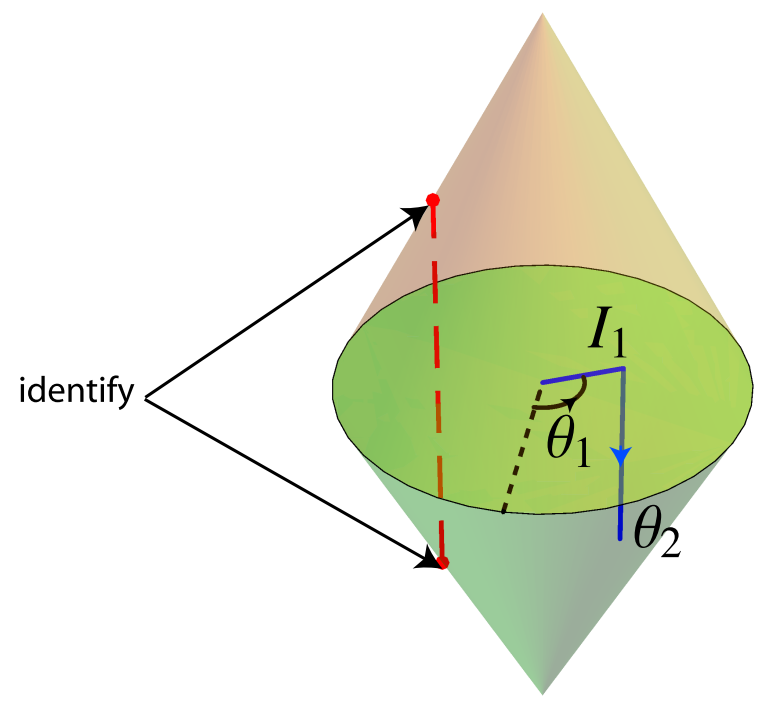

(a) Coordinates on $\mathcal{N}$

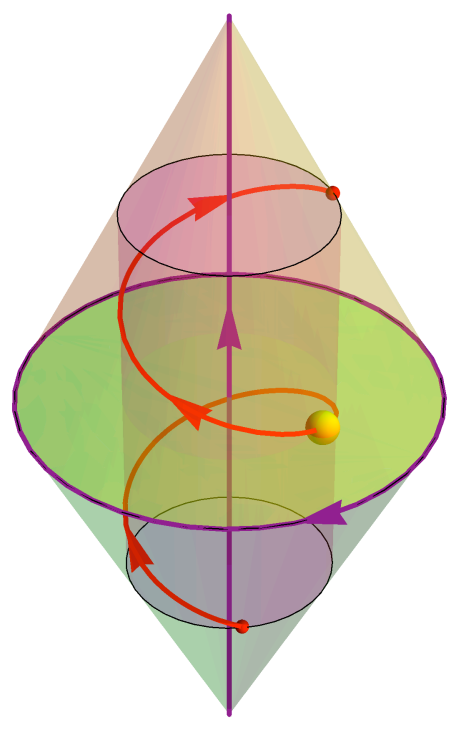

(b) An orbit on $\mathcal{N}$

Figure 1. A model of $\mathcal{N}$

The geometric model of $\mathcal{N}$ is given by two solid cones with points on the boundary cones identified as shown in Figure 1(a). Through each point in the open unit disk with coordinates $I_{1}, \theta_{1}$ there is a line segment (the red dashed line) perpendicular to the disk. The angular coordinate $\theta_{2}$ is measured downward on this segment: $\theta_{2}=0$ is the disk, $\theta_{2}=-\pi$ is the upper boundary cone, and $\theta_{2}=+\pi$ is the lower boundary cone. Each point on the upper boundary cone with coordinates $I_{1}, \theta_{1}, \theta_{2}=-\pi$ is identified with the point on the lower boundary cone with coordinates $I_{1}, \theta_{1}, \theta_{2}=+\pi$. This is our model for the sphere $\mathcal{N}$.

There are two special orbits in this model. The first one is right up the center where $I_{1}=0$ which is periodic with period $2 \pi / p$ and the second one is around the edge where $I_{1}=1 / q$ or $I_{2}=0$ which is periodic with period $2 \pi / q$. Both of them are drawn in purple in Figure 1(b). All the others wrap around a torus where $I_{1}=I_{0}$ and $I_{0}$ is a constant such that $0<I_{0}<1 / q$, as illustrated in Figure $1(\mathrm{~b})$. These orbits hit the open disk where $\theta_{2}=0$ in one point, drawn in yellow in Figure 1(b). These solutions have period $2 \pi$ if $\operatorname{gcd}(q, p)=1$ and are torus knots. 
Sections in $\mathcal{N}$. We select two sections to the flow defined by Hamiltonian (5). Each of them will be a 2-dimensional symplectic submanifold diffeomorphic to a closed disk and such that each orbit intersects at least one of the cross sections and maybe more than once. Refer to Figure 2.

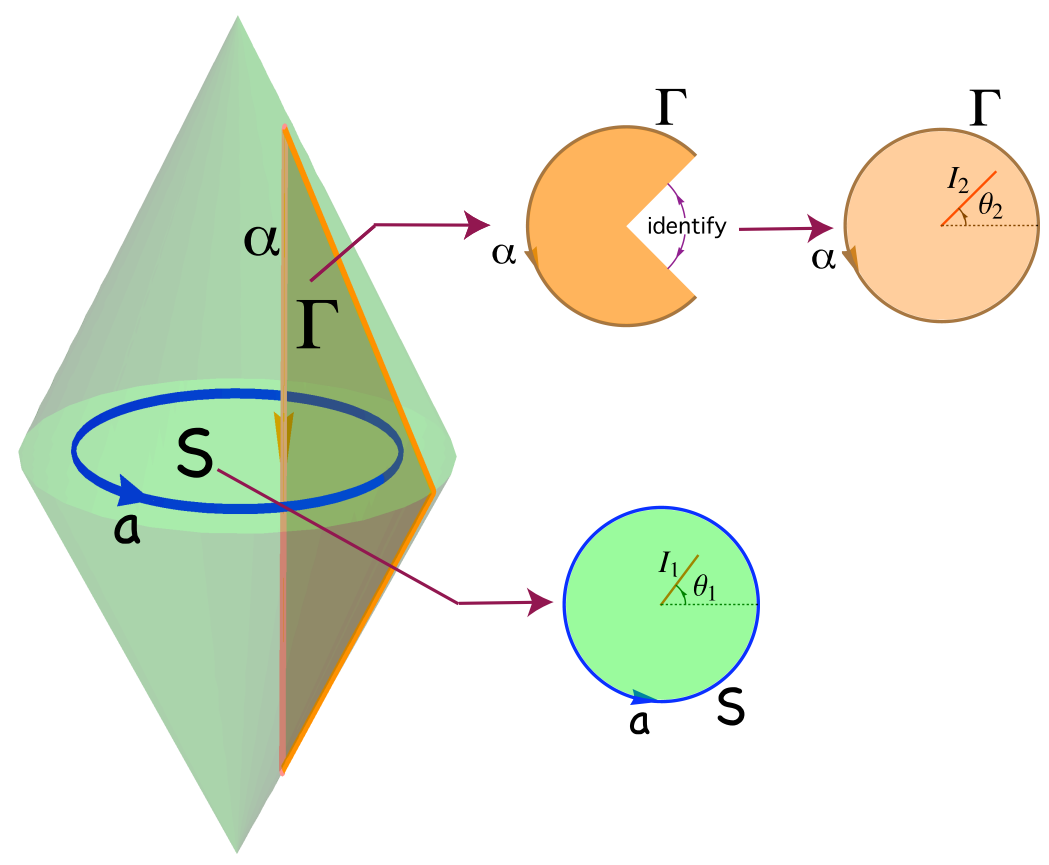

FiguRE 2. Sections in $\mathcal{N}$

The first section, $S$, is the disk where $\theta_{2}=0$ and $0 \leq I_{1} \leq 3 /(4 q)$ and all $\theta_{1}$. Denote the boundary of $S$ by the oriented circle $a$. The section $S$ is a symplectic submanifold of dimension 2 with action-angle coordinates $\left(I_{1}, \theta_{1}\right)$. Orbits cross $S$ upwardly since $\dot{\theta}_{2}=-p$ and $\theta_{2}$ increases downwardly.

The second section, $\Gamma$, is the disk where $\theta_{1}=0$ and $1 /(4 q) \leq I_{1} \leq 1 / q$. At first sight $\Gamma$, the region bounded by the orange curve on the left picture of Figure 2, looks like a triangle but when the identification is taken into account as illustrated in the figure it is indeed a disk. Specifically, $\Gamma$ is a symplectic submanifold of dimension 2 with action-angle coordinates $\left(I_{2}, \theta_{2}\right)$. Orbits cross $\Gamma$ since $\dot{\theta}_{1}=-q$. Denote the boundary of $\Gamma$ by the oriented circle $\alpha$. Here one sees the reason why we measure $\theta_{2}$ downward, because with that convention $I_{2}, \theta_{2}$ are oriented to be symplectic coordinates in $\Gamma$.

Due to the overlap of these two sections every orbit crosses one or the other or both.

Regular Reduction: $q=p=1$. Now we illustrate that $\mathbb{O}$ is a symplectic sphere when $q=p=1$ thus illustrating Reeb's Theorem [50,61]. All solutions are $2 \pi$-periodic and $\theta_{1}$ and $\theta_{2}$ both decrease by one full revolution in time $2 \pi$. In our figures the orbit is a left handed helix traversed upward.

Symplectic maps of 2-dimensional surfaces preserve area and orientation. We will take artistic liberties with area, but be slaves to the orientation. At the top of Figure 3 are the two sections $S$ and $\Gamma$ with their boundary curves $a$ and $\alpha$. Because the two sections overlap, the forward image of $a$ meets $\Gamma$ in an oriented circle $a^{\prime}$ as illustrated, and similarly the forward image of $\alpha$ meets $S$ in an oriented circle $\alpha^{\prime}$. Since $\theta_{2}-\theta_{1}$ is constant in this case the curves are oriented as illustrated.

The flow maps the band between $\alpha$ and $a^{\prime}$ onto the band between $\alpha^{\prime}$ and $a$ symplectically. (We recall that section maps are symplectic.) The point and its image are on the same orbit and so should be identified to form the quotient space $\mathbb{O}$. Thus we have shown that $\mathbb{O}$ is a 2 -sphere with symplectic coordinates.

Singular Reduction: $q=2, p=3$. Now we illustrate that $\mathcal{N}$ is a symplectic orbifold when $q=2, p=3$. The two sections are slightly different now so a little more care is needed. 


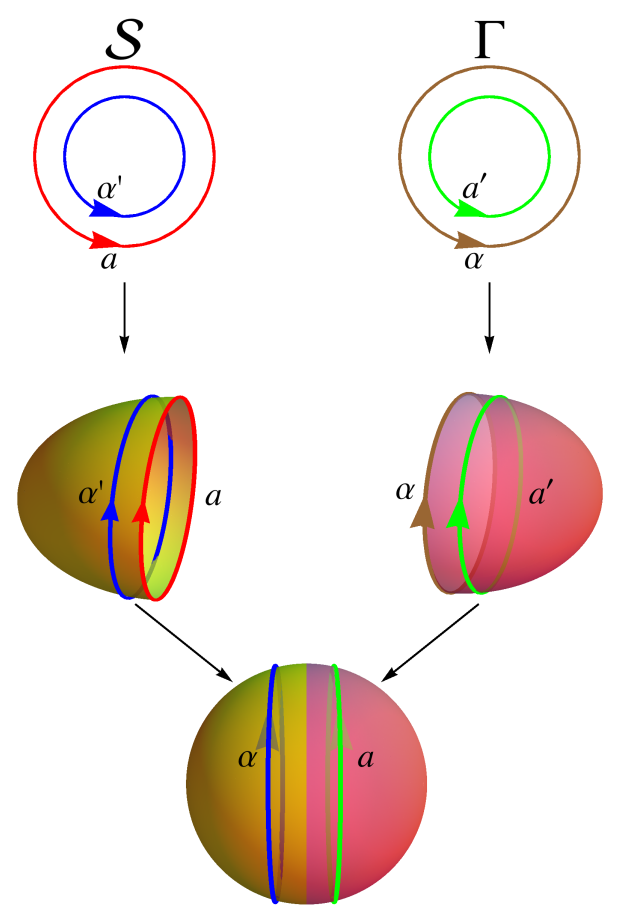

Figure 3. Gluing sections when $q=p=1$

The section $S$ is defined by $\theta_{2}=0$ and has coordinates $I_{1}, \theta_{1}$. The first return time is $T=2 \pi / 3$ and the section map is

$$
I_{1} \mapsto I_{1}, \quad \theta_{1} \mapsto \theta_{1}-4 \pi / 3 .
$$

A point not at the origin hits $S$ three times and repeats. That is

$$
\theta_{1} \mapsto \theta_{1}-4 \pi / 3 \mapsto \theta_{1}-8 \pi / 3 \mapsto \theta_{1}-12 \pi / 3 \equiv \theta_{1} .
$$

Thus an orbit other than $I_{1}=0$ hits $S$ three times equally spaced in $\theta_{1}$, so we should take the sector $0 \leq \theta_{1} \leq 2 \pi / 3$ and identify the lines $\theta_{1}=0$ and $\theta_{1}=2 \pi / 3$ as shown on the left-hand side of Figure 4 to get a sharp cone.

Similarly the section $\Gamma$ is defined by $\theta_{1}=0$ and has coordinates $I_{2}, \theta_{2}$. The first return time is $T=\pi$ and the section map is

$$
I_{2} \mapsto I_{2}, \quad \theta_{2} \mapsto \theta_{2}-\pi .
$$

A point not at the origin hits $S$ two times and repeats. That is

$$
\theta_{2} \mapsto \theta_{2}-\pi \mapsto \theta_{2}-2 \pi \equiv \theta_{2} .
$$

Thus an orbit other than $I_{2}=0$ hits $\Gamma$ two times equally spaced in $\theta_{2}$, so we should take the sector $0 \leq \theta_{2} \leq \pi$ and identify the lines $\theta_{2}=0$ and $\theta_{2}=\pi$ as shown on the right-hand side of Figure 4 to get a cone.

The boundary curves $a, \alpha, a^{\prime}, \alpha^{\prime}$ play the same role in identifying the two parts as in the previous case, see Figure 4.

\section{Integrable and Maximally Super-Integrable Systems}

4.1. Orbit Spaces and Invariants for $n$ Degrees of Freedom. Again let $\mathcal{H}: \mathcal{M} \rightarrow \mathbb{R}$ be a smooth Hamiltonian on a symplectic manifold $\mathcal{M}$ of dimension $2 n$. An integral or invariant for the system is a smooth function $I: \mathcal{M} \rightarrow \mathbb{R}$ such that $\{\mathcal{H}, I\}=0$ where $\{\cdot, \cdot\}$ is the Poisson bracket. An integral $I$ is constant along the solutions of the Hamiltonian system defined by $\mathcal{H}$. Two integrals $I_{1}, I_{2}$ are said to be in involution if $\left\{I_{1}, I_{2}\right\}=0$. The system defined by $\mathcal{H}$ is integrable if there exist $n$ independent integrals in 


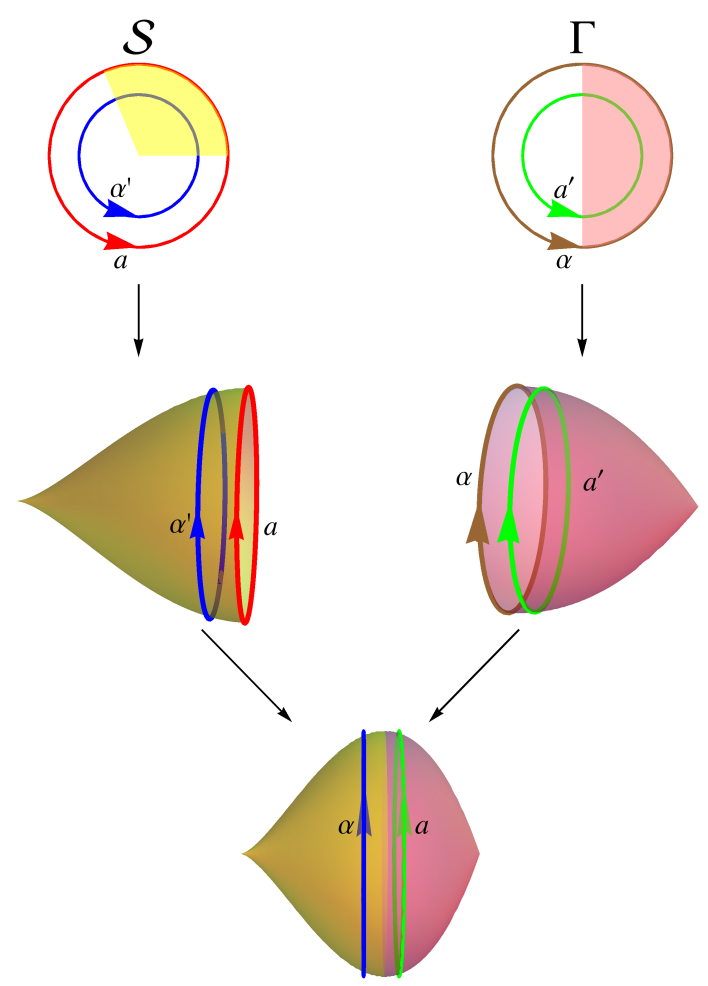

Figure 4. Gluing sections when $q=2, p=3$

involution. A fact usually attributed to Arnold is that an integrable system is foliated almost everywhere by $n$-dimensional invariant tori and cylinders upon which one can find action-angle coordinates [37].

The system defined by $\mathcal{H}$ is super-integrable since it has more than $n$ independent integrals, in fact it is maximally super-integrable as it has $2 n-1$ independent integrals. The number $2 n-1$ is the maximal number of independent integrals a non-trivial $\mathcal{H}$ can have since holding $2 n-1$ independent integrals fixed would define a solution curve in the $2 n$-dimensional space $\mathcal{M}$. The classical example is the Kepler problem which has in addition to energy and angular momentum, that are common to all central force problems, the vector which fixes the pericenter. In general integrable systems are rare and amongst integrable systems super-integrable systems are rarer still — see [37].

Return to the discussion of the Hamiltonian $\mathcal{H}_{R}$ in Section 2. We see that this system is integrable as $I_{1}, I_{2}, \ldots, I_{n}$ are $n$ independent integrals in involution, i.e. $\left\{I_{j}, I_{m}\right\}=0$. Actually, the use of action-angle variables makes obvious it is a maximally super-integrable system since there are $2 n-1$ independent integrals, namely

$$
I_{1}, I_{2}, \ldots, I_{n}, k_{1} \theta_{n}-k_{n} \theta_{1}, k_{1} \theta_{n-1}-k_{n-1} \theta_{1}, \ldots, k_{1} \theta_{2}-k_{2} \theta_{1} .
$$

We return to rectangular coordinates by being mindful of the d'Alembert character [43] and defining

$$
\begin{aligned}
a_{1} & =I_{1}=x_{1}^{2}+y_{1}^{2}, \quad a_{2}=I_{2}=x_{2}^{2}+y_{2}^{2}, \quad \ldots, \quad a_{n}=I_{n}=x_{n}^{2}+y_{n}^{2}, \\
a_{n+1} & =a_{n}^{\left|k_{1}\right| / 2} a_{1}^{\left|k_{n}\right| / 2} \cos \left(k_{1} \theta_{n}-k_{n} \theta_{1}\right)=\operatorname{Re}\left[\left(x_{n}+\operatorname{sgn}\left(k_{1}\right) y_{n} \mathrm{i}\right)^{\left|k_{1}\right|}\left(x_{1}-\operatorname{sgn}\left(k_{n}\right) y_{1} \mathrm{i}\right)^{\left|k_{n}\right|}\right], \\
a_{n+2} & =a_{n}^{\left|k_{1}\right| / 2} a_{1}^{\left|k_{n}\right| / 2} \sin \left(k_{1} \theta_{n}-k_{n} \theta_{1}\right)=\operatorname{Im}\left[\left(x_{n}+\operatorname{sgn}\left(k_{1}\right) y_{n} \mathrm{i}\right)^{\left|k_{1}\right|}\left(x_{1}-\operatorname{sgn}\left(k_{n}\right) y_{1} \mathrm{i}\right)^{\left|k_{n}\right|}\right], \\
\vdots & \\
a_{3 n-3} & =a_{2}^{\left|k_{1}\right| / 2} a_{1}^{\left|k_{2}\right| / 2} \cos \left(k_{1} \theta_{2}-k_{2} \theta_{1}\right)=\operatorname{Re}\left[\left(x_{2}+\operatorname{sgn}\left(k_{1}\right) y_{2} \mathrm{i}\right)^{\left|k_{1}\right|}\left(x_{1}-\operatorname{sgn}\left(k_{2}\right) y_{1} \mathrm{i}\right)^{\left|k_{2}\right|}\right], \\
a_{3 n-2} & =a_{2}^{\left|k_{1}\right| / 2} a_{1}^{\left|k_{2}\right| / 2} \sin \left(k_{1} \theta_{2}-k_{2} \theta_{1}\right)=\operatorname{Im}\left[\left(x_{2}+\operatorname{sgn}\left(k_{1}\right) y_{2} \mathrm{i}\right)^{\left|k_{1}\right|}\left(x_{1}-\operatorname{sgn}\left(k_{2}\right) y_{1} \mathrm{i}\right)^{\left|k_{2}\right|}\right] .
\end{aligned}
$$


Clearly $a_{1} \geq 0, a_{2} \geq 0, \ldots, a_{n} \geq 0$ and the familiar identity $\cos ^{2} \phi+\sin ^{2} \phi=1$ yields

$$
\begin{aligned}
a_{n+1}^{2}+a_{n+2}^{2} & =a_{n}^{\left|k_{1}\right|} a_{1}^{\left|k_{n}\right|}, \\
\vdots & \\
a_{3 n-3}^{2}+a_{3 n-2}^{2} & =a_{2}^{\left|k_{1}\right|} a_{1}^{\left|k_{2}\right|} .
\end{aligned}
$$

Specifying the integrals $a_{1}, a_{2}, \ldots, a_{3 n-2}$ subject to the constraints given above uniquely specifies an orbit because the constraint $a_{n+1}^{2}+a_{n+2}^{2}=a_{n}^{\left|k_{1}\right|} a_{1}^{\left|k_{n}\right|}$ allows one to solve for $\cos \left(k_{1} \theta_{n}-k_{n} \theta_{1}\right)$ and $\sin \left(k_{1} \theta_{n}-k_{n} \theta_{1}\right)$ and hence to find the angle $k_{1} \theta_{n}-k_{n} \theta_{1}$. Similarly the angles $k_{1} \theta_{n-1}-k_{n-1} \theta_{1}, \ldots, k_{1} \theta_{2}-k_{2} \theta_{1}$ are found.

We will call the polynomial invariants $a_{1}, \ldots, a_{3 n-2}$ in (7) a basis set of invariants. Fixing the energy level $\mathcal{H}_{R}=h$ we also get the additional constraint $k_{1} a_{1}+\cdots+k_{n} a_{n}=h$. The invariants $a_{j}, j=1, \ldots, 3 n-2$ together with the $n$ constraints and the inequalities $a_{j} \geq 0, j=1, \ldots, n$ define the orbit space $\mathbb{O}$, thus one can think of the $a_{j}$ 's as the coordinates of the $(2 n-2)$-dimensional orbifold $\mathbb{O}$ embedded in $\mathbb{R}^{3 n-2}$.

Consider the geometry of the orbit space. When $k_{j}=1$ for all $j$ then $\mathbb{O}$ is diffeomorphic to $\mathbb{C P}^{n-1}$ provided $h>0$. However when there is at least one pair $k_{i}, k_{j}$ with $k_{i} \neq k_{j}$ but where $k_{j}>0$ for all $j$, the orbit space for $h>0$ is bounded, but has different types of singularities as we shall see in Section 4.2. In this case the orbifold $\mathbb{O}$ is still homeomorphic to $\mathbb{C P}^{n-1}$, more specifically it has the structure of a weighted complex projective space [4] with weights $k_{j}$ 's. On the other hand if the $k_{j}$ 's have different signs, that is, when $\mathcal{H}_{R}$ in (1) is an indefinite quadratic form, the orbifold $\mathbb{O}$ is not compact.

Proposition 4.1. A Hamiltonian in total resonance (1) is integrable since $I_{1}, \ldots, I_{n}$ are $n$ integrals in involution and in fact it is maximally super-integrable since there are $2 n-1$ independent integrals (6). In this case there are $3 n-2$ polynomial invariants (7) subject to $n-1$ constraints (8).

A model for the orbit space $\mathbb{O}$ is the semi-algebraic variety in $\mathbb{R}^{3 n-2}$ generated by the invariants $\left\{a_{1}, \ldots\right.$, $\left.a_{3 n-2}\right\}$ introduced in (7) where $a_{1} \geq 0, \ldots, a_{n} \geq 0, \mathcal{H}_{R}=k_{1} a_{1}+\cdots+k_{n} a_{n}=h$ and (8) holds, therefore $\left(\mathbb{O}\right.$ is realized by a set of dimension $2 n-2$. When $\mathcal{H}_{R}$ in (1) is a definite quadratic form, $\mathbb{O}$ is a weighted complex projective space, it is compact and homeomorphic to $\mathbb{C P}^{n-1}$ and it is diffeomorphic to it if $k_{j}=1$ for all $j$ and $h>0$. When $\mathcal{H}_{R}$ is indefinite the orbifold $\mathbb{O}$ is unbounded.

One cannot expect more. Consider the Hamiltonian $\mathcal{H}_{R}=\omega_{1} I_{1}+\omega_{2} I_{2}$ written in action-angle variables with $\omega_{1} / \omega_{2}$ irrational. This Hamiltonian is integrable since $I_{1}, I_{2}$ are two independent integrals in involution. Fixing $I_{1}$ and $I_{2}$ defines a 2 -torus $\mathbb{T} \subset \mathbb{R}^{4}$ and the equations on this torus are $\dot{\theta}_{1}=-\omega_{1}, \dot{\theta}_{2}=-\omega_{2}$ with orbits that are dense on $\mathbb{T}$ [43]. Thus any continuous integral must be constant on $\mathbb{T}$ and therefore a function of $I_{1}, I_{2}$. Therefore this Hamiltonian is integrable but not super-integrable.

Resonances in Hamiltonian systems with three or more degrees of freedom have received also the attention of many authors from the point of view of the determination of periodic solutions and their possible bifurcation, reduction theory through polynomial invariants, existence of chaos and other related topics, see for instance $[58,59,31,34,24,23,30,21,17,52]$. In this case the dimension of the orbit space is at least four and the theory turns out rather cumbersome.

One of the issues when $n>2$ is the number of invariants needed in order to treat a specific resonance. Concretely, given a Hamiltonian with unperturbed part (1) with its higher order terms in normal form with respect to $\mathcal{H}_{R}$, the invariants of a basis set are not enough to write down the Hamiltonian as a polynomial of them. For instance, the Poisson brackets among the invariants (7) cannot be represented as polynomials in the $a_{j}$ 's. Indeed one needs to determine a Hilbert basis related to (1), that is, a finite set of invariant polynomials, such that every invariant polynomial may be written as a polynomial function of these basis elements, see [7]. In particular, Egilsson [14] has proved that the minimal size possible for a Hilbert basis associated to a Hamiltonian like (1) is $n^{2}$. Thus the theory for Hamiltonians with $n>2$ requires a special consideration. We shall relegate its study to [46], where we will provide an efficient algorithm due to Derksen and Kemper [11] to compute a Hilbert basis of invariants for a given $\mathcal{H}_{R}$. We will also discuss the treatment of resonant Hamiltonian systems with small perturbations, starting from the reduced Hamiltonian written in terms of the invariants of the Hilbert basis.

4.2. Plateau, Peaks and Ridges. For our perturbation analysis that we shall perform in Sections 6 and 7 we need to consider various types of subsets of $\mathbb{O}$. 
The plateau $L \subseteq \mathbb{O}$ consists of all those points $d \in \mathbb{O}$ such that if $z \in \Pi^{-1}(d)$ then the minimal period through $z$ is $2 \pi$. The only solution to $Z(t) z=z$ is $t=0 \bmod 2 \pi$ and so the action $\mathcal{Z}$ is free at $d$, so locally free. Since $Z(2 \pi)$ is the identity matrix all the characteristic multipliers of the solution through $z$ are +1 . Clearly $L$ is an open subset and it inherits a symplectic structure from $\mathcal{M}$ just as in Reeb's Theorem $[50,61]$ or in regular reduction theory [40], see also [38]. The plateau maybe the whole orbifold as in the case of regular reduction or it may exclude some of the structures given below. In any case the plateau always contains the image under $\Pi$ of all the points $\mathbb{R}^{2 n}$ where $I_{j} \neq 0$ for all $j=1, \ldots, n$.

To understand the substructures of $\mathbb{O}$ we first look at some subsets of $\mathbb{R}^{2 n}$ and establish some notation. Let $D=\{1,2, \ldots, n\}$ be the set of indexes, $F \subseteq D$ and $F^{\dagger}$ the complement of $F$ in $D$, so $D=F \cup F^{\dagger}$. Now let

$$
\mathbb{I}_{F}=\left\{z=\left(x_{1}, \ldots, x_{n}, y_{1}, \ldots, y_{n}\right) \in \mathbb{R}^{2 n}: I_{j}=x_{j}^{2}+y_{j}^{2}=0 \text { for all } j \in F^{\dagger}\right\} .
$$

The set $\mathbb{I}_{F}$ is an invariant symplectic linear subspace of $\mathbb{R}^{2 n}$ of dimension $2 f$ where $f$ is the number of elements in $F$. It is filled with periodic solutions. For example, taking $F=D$ one has $\mathbb{I}_{D}=\mathbb{R}^{2 n}$, $\mathbb{I}_{D^{\dagger}}$ is the origin in $\mathbb{R}^{2 n}$. For each of these linear spaces one can perform the reduction as previously defined by fixing $\mathcal{H}=h$ where $h$ is a regular value and identifying orbits to a point to obtain an orbifold $\mathbb{O}_{F}$ of dimension $2 f-2$. Thus $\mathbb{O}$ is a complicated union of suborbifolds. The various periods of the solutions in $\mathbb{O}$ single out which of these suborbifolds will be interesting for perturbation analysis.

If $F=\{s\}$ then $\mathbb{I}_{F}=\left\{z=\left(0, \ldots, 0, x_{s}, 0, \ldots, 0, y_{s}, 0, \ldots, 0\right)\right\}$ is a 2-dimensional linear subspace filled with periodic solutions of period $T=2 \pi /\left|k_{s}\right|$ which is traditionally called a normal mode. The image of this linear subspace under $\Pi$ is a single point $P_{s} \in \mathbb{O}$ and it is a peak if $\left|k_{s}\right|>1$ and $k_{i} / k_{s}$ is not an integer when $i \neq s$. The maximum number of peaks in $\mathbb{O}$ is $n$. All the nonzero solutions in $\mathbb{I}_{F}$ are periodic with least period $T$ and multipliers $e^{ \pm k_{1} T \mathrm{i}}, e^{ \pm k_{2} T \mathrm{i}}, \ldots, e^{ \pm k_{n} T \mathrm{i}}$ where $e^{ \pm k_{s} T \mathrm{i}}=1$ while all the other multipliers are different from one. The only solutions of $Z(t) z=z$ are $t=0,2 \pi /\left|k_{s}\right|, 2\left(2 \pi /\left|k_{s}\right|\right), \ldots,\left(\left|k_{s}\right|-1\right)\left(2 \pi /\left|k_{s}\right|\right)$ $\bmod 2 \pi$ and so the action $\mathcal{Z}$ is locally free at this point.

A ridge is in the complement of the plateau and the peaks. Let $F=\left\{s_{1}, \ldots, s_{m}\right\}$ be a proper subset of $D$, so $1<m<n$. The linear space $\mathbb{I}_{F}$ has dimension $2 m$ and the suborbifold $\mathbb{O}_{F}$ has dimension $2 m-2$. A ridge is the subset $\mathbb{G}_{F} \subseteq \mathbb{O}_{F}$ formed by the points $d \in \mathbb{O}_{F}$ such that $z \in \Pi^{-1}(d)$ has action-angle coordinates with $I_{s_{1}} \neq 0, \ldots, I_{s_{m}} \neq 0$ and all the other $I_{j}$ 's are zero, $z$ is filled with periodic solutions of least period $T=2 \pi / \kappa$ with $\kappa=\operatorname{gcd}\left(\left|k_{s_{1}}\right|, \ldots,\left|k_{s_{m}}\right|\right)>1$ such that $2(n-m)$ characteristic multipliers are different from one. The solutions of $Z(t) z=z$ are $t=0,2 \pi / \kappa, \ldots,(\kappa-1)(2 \pi / \kappa) \bmod 2 \pi$, thence the action $\mathcal{Z}$ is locally free at this point.

Since each point falls into one of the above categories the action $\mathcal{Z}$ defined in (2) is locally free at every point.

For insight consider the case when all the $k_{i}$ 's are positive so $\mathcal{H}_{R}=h$ with $h=1$ is an ellipsoid or a topological sphere of dimension $2 n-1$. For $n=2$ the flow on the 3 -sphere was discussed above. But a ridge can only occur for systems of at least three degrees of freedom where the integral manifold is at least 5 -dimensional and the orbit space is at least 4-dimensional. For higher dimensions we need a more symbolic representation. To this end return to action-angle variables and project $\mathcal{N}$ onto $\left(I_{1}, \ldots, I_{n}\right)$-space as follows,

$$
\mathbb{P}: \mathcal{N} \rightarrow \mathbb{S}:\left(I_{1}, \ldots, I_{n}, \theta_{1}, \ldots, \theta_{n}\right) \mapsto\left(I_{1}, \ldots, I_{n}\right)
$$

where

$$
\mathbb{S}=\left\{\left(I_{1}, \ldots, I_{n}\right) \in \mathbb{R}^{n}: k_{1} I_{1}+\cdots+k_{n} I_{n}=1, I_{j} \geq 0\right\} .
$$

$\mathbb{S}$ is the simplex illustrated in Figure 5 for $n=2,3$. In this figure each point in the simplex represents a torus $\mathbb{T}^{j}$, the $j$-dimensional torus (the circle is the 1-torus). That is, if $d \in \mathbb{S}$ then $\mathbb{P}^{-1}(d) \in \mathcal{N}$ is a torus and we say the torus $\mathbb{P}^{-1}(d)$ is above $d$. Above each vertex is a circle $\mathbb{T}^{1}$, above each edge is a 2 -torus $\mathbb{T}^{2}$ and above each interior point of a triangle is a 3 -torus $\mathbb{T}^{3}$. This simplistic representation does not tell us just how these tori are attached to each other, so we refer the reader to the beautiful theory found in Fomenko et al. [22].

For $n=2$ refer to the image on the left in Figure 5. If $k_{1}=k_{2}=1, \operatorname{gcd}\left(k_{1}, k_{2}\right)=1$ then every point is in the plateau, i.e. the orbit space is regular. If $k_{1}=1, k_{2}=2, \operatorname{gcd}\left(k_{1}, k_{2}\right)=1$ then above $\left(0,1 / k_{2}\right)$ is a peak and all the other points are in the plateau. If $k_{1}=2, k_{2}=3, \operatorname{gcd}\left(k_{1}, k_{2}\right)=1$ then above $\left(0,1 / k_{2}\right)$ and $\left(1 / k_{1}, 0\right)$ are peaks and all the other points are in the plateau, i.e. the orbit space has two singular points. 


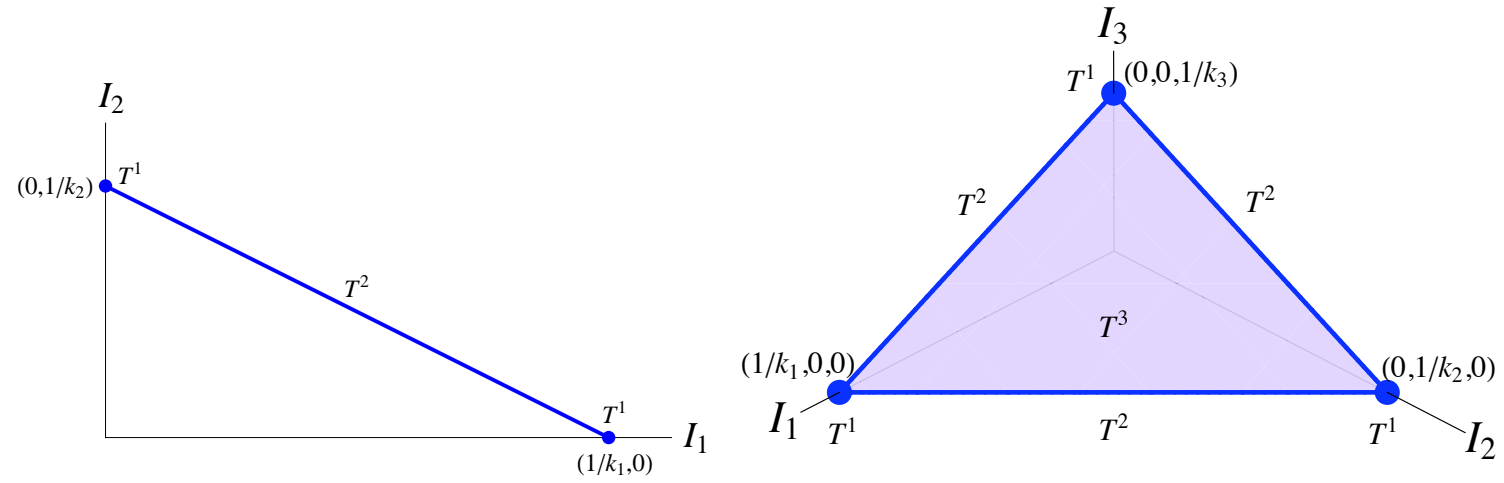

FiguRE 5 . The simplex $\mathbb{S}$ for $n=2$ (left) and $n=3$ (right)

For $n=3$ refer to the image on the right in Figure 5. If $k_{1}=2, k_{2}=3, k_{3}=5$ then $\operatorname{gcd}\left(k_{1}, k_{2}, k_{3}\right)=$ $\operatorname{gcd}\left(k_{1}, k_{2}\right)=\operatorname{gcd}\left(k_{2}, k_{3}\right)=\operatorname{gcd}\left(k_{3}, k_{1}\right)=1$ and the vertices correspond to peaks and all the other points are in the plateau (even the edges). If $k_{1}=6, k_{2}=10, k_{3}=15$ then $\operatorname{gcd}\left(k_{1}, k_{2}, k_{3}\right)=1, \operatorname{gcd}\left(k_{1}, k_{2}\right)=$ $2, \operatorname{gcd}\left(k_{2}, k_{3}\right)=5, \operatorname{gcd}\left(k_{3}, k_{1}\right)=3$ then the vertices correspond to peaks, the edges correspond to ridge points, and the interior points are in the plateau. If $k_{1}=1, k_{2}=2, k_{3}=3$ then $\operatorname{gcd}\left(k_{1}, k_{2}, k_{3}\right)=1, \operatorname{gcd}\left(k_{1}, k_{2}\right)=$ $1, \operatorname{gcd}\left(k_{2}, k_{3}\right)=1, \operatorname{gcd}\left(k_{3}, k_{1}\right)=1$, the vertex that corresponds to $k_{1}$ is not a peak but is a plateau point whereas the other two vertices are peaks. The rest of points are in the plateau.

Any solution of the Hamiltonian system (1) is periodic with period $T=2 \pi / g$ where $g$ is a positive integer. Let $z \in \mathbb{R}^{2 n}, z \neq 0$, so as we have seen above the only solutions of $Z(t) z=z$ are $t=0, T, 2 T, \ldots,(g-1) T$. This means that the isotropy subgroup of $\mathcal{Z}$ is non-trivial for all nonzero $z \in \mathbb{R}^{2 n}$. Thus we have a well defined orbifold with regular points at the plateau and singularities at the peaks and ridges.

The only exception to the classification made above occurs when the Hamiltonian (1) is indefinite. Consider the solutions passing though a point $P_{s} \in \mathbb{O}$ with $x_{j}=y_{j}=0$ if $j \neq s$ and take the level set $h=0$. Then the only possible solution to the system related to (1) is $z=0$, hence $x_{s}=y_{s}=0$. The isotropy subgroup of $\mathcal{Z}$ is trivial for all $z \in \mathbb{R}^{2 n}$. This gives rise to the point $\left(a_{1}, \ldots, a_{n}, \ldots, a_{3 n-2}\right)=(0, \ldots, 0, \ldots, 0)$ on $\mathbb{O}$ which is a singularity in the orbifold but different from a peak or a ridge. Its isotropy subgroup of $\mathcal{Z}$ is trivial, so $P_{s}$ is related to a unique point of $\mathbb{R}^{2 n}$, which is the origin of this space. For $n=2$ see Section 4.3.

4.3. Orbits Spaces and Invariants for the $q: p$ Resonance. One of our main goals is the study of the unfolding of resonant systems subject to small perturbations using normalization and invariants. Let us consider again the two degrees of freedom system $\mathcal{H}_{q p}$ in (5) of Section 3.2. In this section we give the geometry of the orbit spaces for two degrees of freedom systems in preparation for the bifurcation analysis in Section 7 . This resonance has been treated by many authors from different points of view; see for example $[27,55,32,33,8,16,48,18,28,56,29]$.

From (7) a basis set of invariants associated to the $q: p$ resonance is

$$
\begin{aligned}
& a_{1}=I_{1}=x_{1}^{2}+y_{1}^{2}, \\
& a_{2}=I_{2}=x_{2}^{2}+y_{2}^{2}, \\
& a_{3}=I_{1}^{|p| / 2} I_{2}^{q / 2} \cos \left(q \theta_{2}-p \theta_{1}\right)=\operatorname{Re}\left[\left(x_{1}-\operatorname{sgn}(p) y_{1} \mathrm{i}\right)^{|p|}\left(x_{2}+y_{2} \mathrm{i}\right)^{q}\right], \\
& a_{4}=I_{1}^{|p| / 2} I_{2}^{q / 2} \sin \left(q \theta_{2}-p \theta_{1}\right)=\operatorname{Im}\left[\left(x_{1}-\operatorname{sgn}(p) y_{1} \mathrm{i}\right)^{|p|}\left(x_{2}+y_{2} \mathrm{i}\right)^{q}\right],
\end{aligned}
$$

subject to the constraint

$$
a_{3}^{2}+a_{4}^{2}=a_{1}^{|p|} a_{2}^{q}, \quad a_{1} \geq 0, a_{2} \geq 0 .
$$

Note that $a_{3}$ and $a_{4}$ are polynomials in $x, y$ of degree $q+|p|$.

The Poisson brackets associated to the invariants are computed using the relations of the $a_{j}$ 's in terms of the action-angle coordinates $I_{j}$ 's, $\theta_{j}$ 's and then transformed back to the invariants. The explicit expressions 
are given in Table 1. All Poisson brackets are polynomial in the invariants as $q$ and $p$ are nonzero integers. In fact the invariants (9) form a Hilbert basis.

\begin{tabular}{|c|c|c|c|c|}
\hline$\{\}$, & $a_{1}$ & $a_{2}$ & $a_{3}$ & $a_{4}$ \\
\hline$a_{1}$ & 0 & 0 & $2 p a_{4}$ & $-2 p a_{3}$ \\
$a_{2}$ & 0 & 0 & $-2 q a_{4}$ & $2 q a_{3}$ \\
$a_{3}$ & $-2 p a_{4}$ & $2 q a_{4}$ & 0 & $a_{1}^{|p|-1} a_{2}^{q-1}\left(q^{2} a_{1}-p|p| a_{2}\right)$ \\
$a_{4}$ & $2 p a_{3}$ & $-2 q a_{3}$ & $-a_{1}^{|p|-1} a_{2}^{q-1}\left(q^{2} a_{1}-p|p| a_{2}\right)$ & 0 \\
\hline
\end{tabular}

TABLE 1. Poisson algebra of the invariants $a_{1}, \ldots, a_{4}$. The $a_{i}$ 's of the first column are put in the left-hand side of the bracket, and the ones of the first row are placed on the right-hand side of the brackets

As discussed above, an orbit of the system is uniquely specified by the four invariants subject to the constraints (10) and so the orbit space $\mathbb{O}$ is determined by (9), (10) and

$$
\mathcal{H}_{q p}=q a_{1}+p a_{2}=h .
$$

Solve (11) for $a_{2}$ and substitute into the constraint equation to get

$$
a_{3}^{2}+a_{4}^{2}=a_{1}^{|p|}\left(\frac{h-q a_{1}}{p}\right)^{q}
$$

which defines a surface in $\left(a_{1}, a_{3}, a_{4}\right)$-space. This surface is a representation of the orbit space $\mathbb{O}$. We distinguish different situations according to the values of $q$ and $p$. Note that (12) defines a surface of revolution, so let $\rho, \psi$ be polar coordinates in the $\left(a_{3}, a_{4}\right)$-plane so that equation (12) becomes

$$
\rho^{2}=a_{1}^{|p|}\left(\frac{h-q a_{1}}{p}\right)^{q} .
$$

We will say that the orbit space $\mathbb{O}$ is an orange, turnip or a lemon if the surface (12) is compact and has 0,1 or 2 peaks respectively. In the non-compact case we will say that $\mathbb{O}$ is a cap (yarmulke) if it is smooth or a trumpet if it has a singular point (which may or may not be a peak) of conical type.

Case $p>0$. In this situation let $h>0$. The surface in $\left(a_{1}, a_{3}, a_{4}\right)$-space is compact. See Figure 6 above for the views of the $\left(\rho, a_{1}\right)$-sections of the orbit spaces when $p>0$.

First let us specify $p=q=1$. Then by completing the square we have $\rho^{2}+\left(a_{1}-h / 2\right)^{2}=h^{2} / 4$ which is a circle of radius $h / 2$ centered at $\left(\rho, a_{1}\right)=(0, h / 2)$, and so (13) defines a sphere in $a$-space of radius $h / 2$ and center at $\left(a_{1}, a_{3}, a_{4}\right)=(h / 2,0,0)$, see Figure $6(\mathrm{a})$. Here there are no singularities so this case falls under regular reduction.

Now consider the case where $q$ and $p$ are positive and $q \neq p$, then the right-hand side of equation (13) is positive for $0<a_{1}<h / q$ and the surface of revolution is smooth for $a_{1}$ in that range. But the right-hand side is zero for $a_{1}=0$ and $a_{1}=h / q$ and these are the candidates for singularities. Near $a_{1}=0$ from (13) we see that $\rho \sim c a_{1}^{p / 2}$ where $c>0$ is a constant. Thus the surface of revolution is only smooth at $a_{1}=0$ if $p=1$. For $p>1$ the surface at $a_{1}=0$ has a peak which is cone-like when $p=2$ and is cusp-like for $p>2$. The peak gets sharper for larger $p$. According to Section 4.2 this peak is labelled by $P_{2}$.

Similarly near $a_{1}=h / q$ we see that $\rho \sim c\left(h-q a_{1}\right)^{q / 2}$ where $c>0$ is a constant. Thus the surface of revolution is only smooth at $a_{1}=h / q$ if $q=1$. When $q>1$ the surface has a peak at $a_{1}=h / q$ and the peak gets sharper for larger $q$. The peak is cone-like when $q=2$ and cusp-like for $q>2$. See Figures 6(b) and $6(\mathrm{c})$. This peak is labelled by $P_{1}$. 

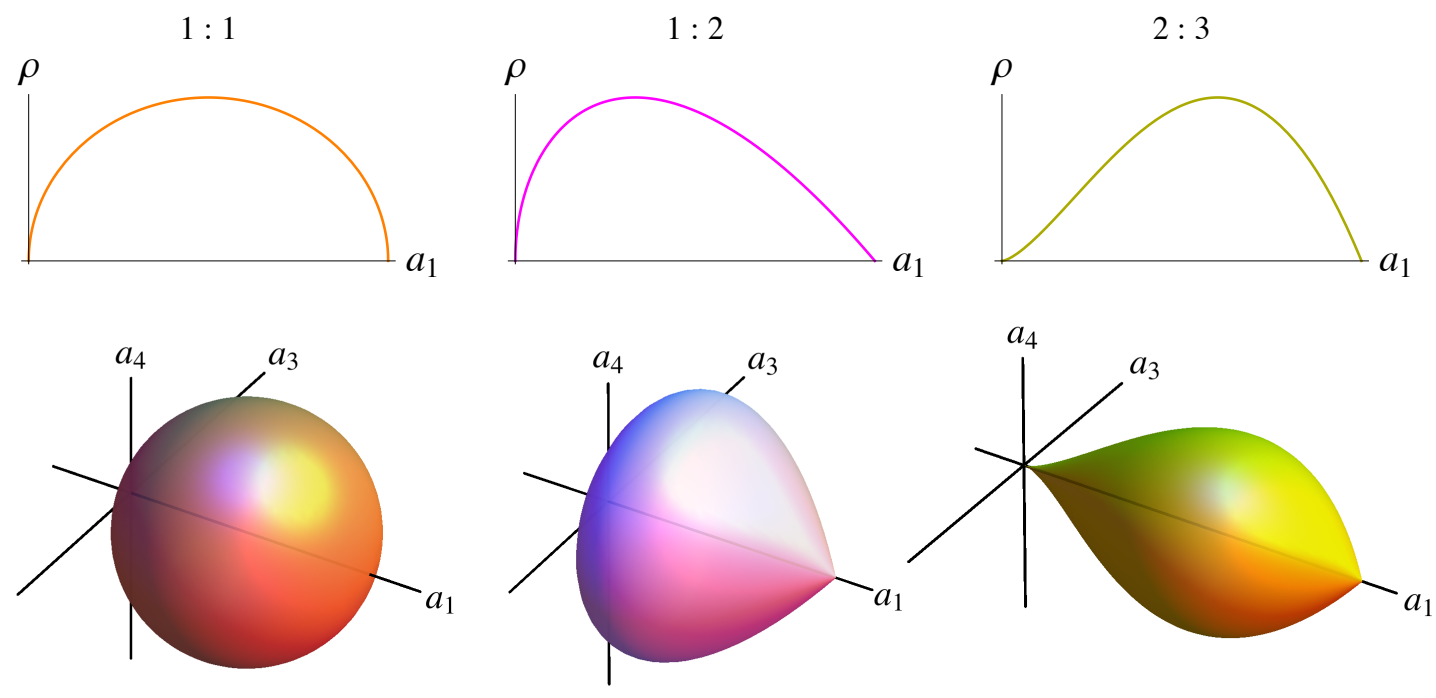

(a) Orange 1:1

(b) Turnip 2:1

(c) Lemon 2:3

Figure 6 . Case $p>0$. Above: $\rho$ versus $a_{1}$. Below: Orbit spaces

$\mathrm{h}<0$
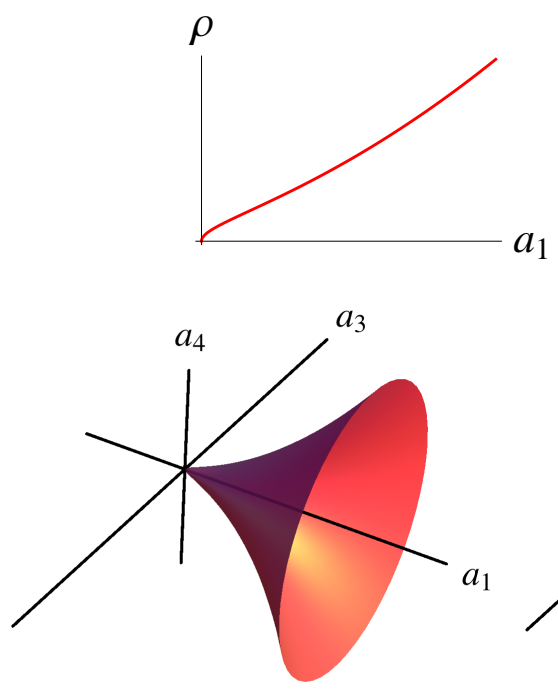

(a) Cap

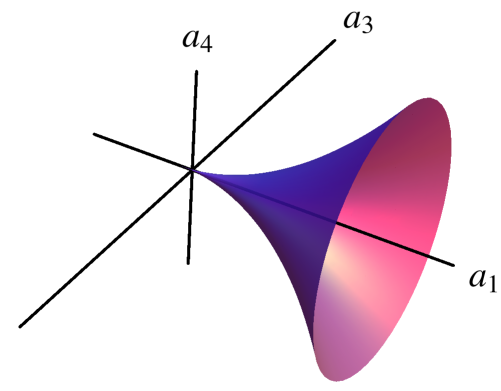

(b) Trumpet (not a peak) $\mathrm{h}>0$
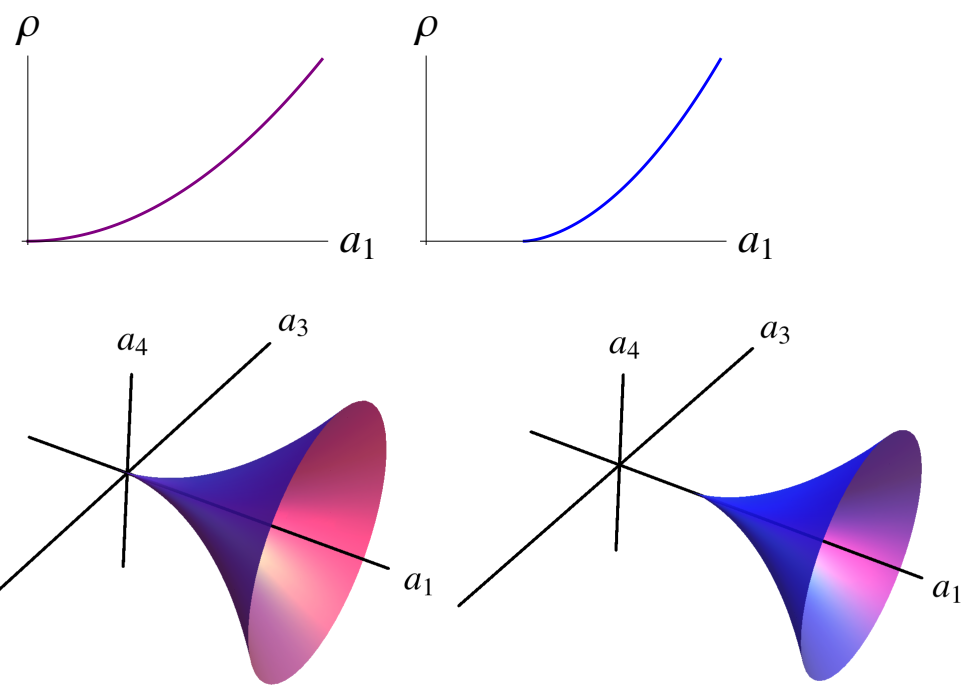

(c) Trumpet (a peak)

Figure 7. Case $q=3, p=-1$. Above: $\rho$ versus $a_{1}$. Below: Orbit spaces

Case $p<0$. The surface of revolution is unbounded and it is smooth when the right-hand side of (12) is positive. As always $a_{1} \geq 0$ but $a_{2} \geq 0$ implies $a_{1} \geq h / q$. See Figure 7 .

When $h<0$ the right-hand side of (12) is zero only at $a_{1}=0$ and nearby $\rho \sim c a_{1}^{|p| / 2}$ with $c$ a positive constant. Thus the surface is smooth, that is, a cap at $a_{1}=0$ when $|p|=1$, it is cone-like when $|p|=2$ and is cusp-like when $|p|>2$, i.e. one gets trumpets. Thus the surface has a peak at $a_{1}=0$ when $|p| \geq 2$. The peak is called $P_{2}$. 
When $h=0$ the right-hand side of (12) is zero at $a_{1}=0$ and nearby $\rho \sim c a_{1}^{(q+|p|) / 2}$ with $c>0$ a constant. Thus the surface is cone-like when $q+|p|=2$ and is cusp-like when $q+|p|>2$, thence it is always a trumpet. So when $h=0$ there is always a conical singularity at $a_{1}=0$. This is the point of $\mathbb{O}$ that corresponds to the origin in $\mathbb{R}^{4}$. It is not a peak nor a ridge, see the last paragraph of Section 4.2 .

When $h>0$ the right-hand side of (12) is zero at $a_{1}=h / q$ and nearby $\rho \sim c\left(q a_{1}-h\right)^{q / 2}$ where $c>0$ is a constant. Thus the surface is smooth, i.e. a cap, at $a_{1}=h / q$ when $q=1$, is cone-like when $q=2$ and is cusp-like when $q>2$. So there is a peak at $a_{1}=h / q$ provided $q \geq 2$ and then the orbifolds $\mathbb{O}$ are trumpets. In this case the peak is named $P_{1}$.

\section{Symplectic Coordinates in the Orbit Space For the $q: p$ Resonance}

5.1. Coordinates for the Regular Points. In order to analyze the stability of equilibria, the possible bifurcations of periodic solutions or the existence of KAM tori, it is convenient to introduce symplectic coordinates valid in neighborhoods of a regular point. We present the procedure for two degrees of freedom and its generalization to $n \geq 2$ will appear in [46]. It is emphasized that as far as we know there is not a systematic approach to build symplectic coordinates around a regular point of the orbit space, thus our procedure is new, although a procedure to get Darboux coordinates in a constructive way using Lie transformations can be seen in [41]. However our approach is more appropriate for resonant Hamiltonians.

Working with action-angle coordinates we build a linear transformation requiring that it is a 1:1 transformation. We also take into account that the occurrence of the angles $\theta_{j}$ 's in the expressions for $a_{3}, a_{4}$ in (9) is through the combination $q \theta_{2}-p \theta_{1}$. Also for a fixed energy $h$ one has $h=q I_{1}+p I_{2}$, so we let $J_{1}=q I_{1}+p I_{2}$ and simplify the calculations a bit by assuming that $J_{2}$ does not depend on $I_{2}$. A slightly different change is derived when $J_{2}$ is considered to be a function of $I_{2}$ as we shall see later. So we start with

$$
\begin{array}{ll}
J_{1}=q I_{1}+p I_{2}, & J_{2}=\alpha I_{1}, \\
\psi_{1}=\beta \theta_{1}+\gamma \theta_{2}, & \psi_{2}=\delta\left(q \theta_{2}-p \theta_{1}\right),
\end{array}
$$

with unknowns $\alpha, \beta, \gamma$ and $\delta$.

The change (14) is symplectic when $\beta=0, \gamma=1 / p$ and $\delta=-1 /(\alpha p)$. Writing down the $a_{k}$ 's in terms of the $J_{k}$ 's, $\psi_{k}$ 's we end up with

$$
\begin{aligned}
& a_{1}=\frac{J_{2}}{\alpha}, \\
& a_{2}=\frac{\alpha J_{1}-q J_{2}}{\alpha p}, \\
& a_{3}=\left(\frac{J_{2}}{\alpha}\right)^{|p| / 2}\left(\frac{\alpha J_{1}-q J_{2}}{\alpha p}\right)^{q / 2} \cos \left(\alpha p \psi_{2}\right), \\
& a_{4}=\left(\frac{J_{2}}{\alpha}\right)^{|p| / 2}\left(\frac{\alpha J_{1}-q J_{2}}{\alpha p}\right)^{q / 2} \sin \left(\alpha p \psi_{2}\right) .
\end{aligned}
$$

In order to get a 1:1 transformation we need that the arguments of the cosine and sine in (15) be $\pm \psi_{2}$. Thus we set $\alpha=1 /|p|$ so $J_{2}=I_{1} /|p|$ and $\psi_{2}=|p|\left[\theta_{1}-(q / p) \theta_{2}\right]$.

Now we are ready to define action-angle coordinates, $J, \psi$, through

$$
J \equiv J_{2}=\frac{I_{1}}{|p|}, \quad \psi \equiv \psi_{2}=|p|\left[\theta_{1}-(q / p) \theta_{2}\right]
$$

noting that $J$ and $\psi$ are well defined for a regular point of (10) and that $\{J, \psi\}=2$. 
Applying (16) to (9), or to (15), and taking into account that $J_{1}=h$, the relation among the invariants $a_{j}$ 's and the action-angle variables $J, \psi$ is

$$
\begin{aligned}
& a_{1}=|p| J \\
& a_{2}=\frac{h-q|p| J}{p}, \\
& a_{3}=(|p| J)^{|p| / 2}\left(\frac{h-q|p| J}{p}\right)^{q / 2} \cos \psi, \\
& a_{4}=-\operatorname{sgn}(p)(|p| J)^{|p| / 2}\left(\frac{h-q|p| J}{p}\right)^{q / 2} \sin \psi .
\end{aligned}
$$

Rectangular coordinates $x, y$ are introduced in terms of $J$ and $\psi$ by means of $J=x^{2}+y^{2}, \psi=\tan ^{-1}(y / x)$, noticing that $\{x, y\}=1$. After a few manipulations we arrive at

$$
\begin{aligned}
& a_{1}=|p|\left(x^{2}+y^{2}\right), \\
& a_{2}=\frac{h-q|p|\left(x^{2}+y^{2}\right)}{p}, \\
& a_{3}=|p|^{|p| / 2} x\left(x^{2}+y^{2}\right)^{(|p|-1) / 2}\left[\frac{h-q|p|\left(x^{2}+y^{2}\right)}{p}\right]^{q / 2}, \\
& a_{4}=-\operatorname{sgn}(p)|p|^{|p| / 2} y\left(x^{2}+y^{2}\right)^{(|p|-1) / 2}\left[\frac{h-q|p|\left(x^{2}+y^{2}\right)}{p}\right]^{q / 2} .
\end{aligned}
$$

Obviously in $\left(a_{1}, x, y\right)$-space the transformed surface is given by the first equation of (18).

If we assume that $J_{2}$ depends only on $I_{2}$ we make

$$
\begin{aligned}
J_{1} & =q I_{1}+p I_{2}, & J_{2} & =\alpha I_{2}, \\
\psi_{1} & =\beta \theta_{1}+\gamma \theta_{2}, & \psi_{2} & =\delta\left(q \theta_{2}-p \theta_{1}\right),
\end{aligned}
$$

and proceeding similarly as above we arrive at the $1: 1$ transformation

$$
\begin{aligned}
& a_{1}=\frac{h-q p\left(x^{2}+y^{2}\right)}{q} \\
& a_{2}=q\left(x^{2}+y^{2}\right), \\
& a_{3}=q^{(q-|p|) / 2} x\left(x^{2}+y^{2}\right)^{(q-1) / 2}\left[h-q p\left(x^{2}+y^{2}\right)\right]^{|p| / 2}, \\
& a_{4}=q^{(q-|p|) / 2} y\left(x^{2}+y^{2}\right)^{(q-1) / 2}\left[h-q p\left(x^{2}+y^{2}\right)\right]^{|p| / 2},
\end{aligned}
$$

which is an alternative to (18).

The inverse of (18) is given by

$$
x=|p|^{-1 / 2} a_{1}^{(1-|p|) / 2} a_{2}^{-q / 2} a_{3}, \quad y=-\operatorname{sgn}(p)|p|^{-1 / 2} a_{1}^{(1-|p|) / 2} a_{2}^{-q / 2} a_{4},
$$

while the inverse of (20) yields

$$
x=q^{-1 / 2} a_{1}^{-|p| / 2} a_{2}^{(1-q) / 2} a_{3}, \quad y=q^{-1 / 2} a_{1}^{-|p| / 2} a_{2}^{(1-q) / 2} a_{4} .
$$

We need to make an extra transformation to get the desired symplectic coordinates around a specific regular point of the surface (10) for a given Hamiltonian in $\mathbb{O}$. If $\left(a_{1}^{0}, a_{3}^{0}, a_{4}^{0}\right)$, together with $a_{2}^{0}=\left(h-q a_{1}^{0}\right) / p$, represents a particular critical point of the equations of motion associated to the Hamiltonian, the change (21) (or (22)) is used to obtain the point $\left(x_{0}, y_{0}\right)$ in the projection defined through (18) (or through (20)). Then, we introduce new coordinates $\bar{x}, \bar{y}$ as $x=\bar{x}+x_{0}, y=\bar{y}+y_{0}$ and perform a Taylor expansion of the Hamiltonian function in terms of $\bar{x}, \bar{y}$ around $\bar{x}=\bar{y}=0$. Since the point of the surface (12) we are analyzing is regular, the transformations (18) and (20) and their respective inverses (21) and (22) have to be analytic in a neighborhood of $\left(x_{0}, y_{0}\right)$. When $p=1$ the change (20) cannot be applied around the point $(0,0,0)$, but one applies instead the transformation (18), while when $q=1$ the change (18) cannot be applied around $(h, 0,0)$ but (20) can be used. In the remaining cases both (18) and (20) and their inverses are analytic. Therefore, 
one can always obtain $\bar{x}, \bar{y}$ as a pair of symplectic rectangular coordinates. When applying either (18) or (20) to a given Hamiltonian we divide the resulting Hamiltonian by 2 since in the change from rectangular to action-angle coordinates we multiplied the transformed Hamiltonian by 2 .

Proposition 5.1. Let $\mathcal{H}$ be a Hamiltonian in $q: p$ resonance defined in the orbit space $\mathbb{O}$ with $q$ and $p$ as in (5). Let $\left(a_{1}^{0}, a_{3}^{0}, a_{4}^{0}\right)$ satisfying (12) represent a regular critical point of the vector field associated to $\mathcal{H}$ and let $\left(a_{1}^{0}, x_{0}, y_{0}\right)$ be the critical point in $\left(a_{1}, x, y\right)$-space where $x_{0}, y_{0}$ are obtained from $\left(a_{1}^{0}, a_{3}^{0}, a_{4}^{0}\right)$ by means of (21) or of (22). The successive changes given by (18) (or (20)) and by $x=\bar{x}+x_{0}, y=\bar{y}+y_{0}$ transform $\mathcal{H}$ into a function in the symplectic rectangular coordinates $\bar{x}$ and $\bar{y}$ in a neighborhood of the critical point $\left(a_{1}^{0}, a_{3}^{0}, a_{4}^{0}\right)$. The surface (12) is transformed into $a_{1}=|p|\left[\left(\bar{x}+x_{0}\right)^{2}+\left(\bar{y}+y_{0}\right)^{2}\right]$ (or into $\left.a_{1}=\left\{h-q p\left[\left(\bar{x}+x_{0}\right)^{2}+\left(\bar{y}+y_{0}\right)^{2}\right]\right\} / q\right)$.

5.2. Symplectic Smoothing. In order to study the eventual singular points appearing in the orbit space defined by (12) and the flow of the harmonic oscillator in $q: p$ resonance (usually with an attached perturbation) around the singular points, we construct a set of symplectic changes of coordinates with the aim of removing the conical singularities on the surface $\mathbb{O}$ and perform local studies around these points. The procedure is presented for arbitrary values of integers $q>0, p \neq 0$ and $\operatorname{gcd}(q, p)=1$. We focus on the singularities occurring at $a_{1}=0$ (the peak $P_{2}$ and the singularity of the origin in $a$-space when $h=0$ ) and at $a_{1}=h / q$ (the peak $P_{1}$ ) whereas the generalization for $n \geq 2$ will appear in [46]. We stress that our analysis covers all possible cases of singular orbits spaces when $n=2$. In addition, although a few particular situations have been studied previously, see for instance [23, 24], our approach is new, straightforward and can be extend to any dimension easily.

In order to unfold the singularity at $a_{1}=0$ we introduce the change

$$
w=a_{3}+a_{4} \mathrm{i}, \quad \bar{w}=a_{3}-a_{4} \mathrm{i}, \quad w \bar{w}=a_{3}^{2}+a_{4}^{2} .
$$

Making $w=z^{|p|}$ the constraint (12) is transformed into

$$
z^{|p|} \bar{z}^{|p|}=a_{1}^{|p|}\left(\frac{h-q a_{1}}{p}\right)^{q},
$$

whence one gets

$$
z \bar{z}=a_{1}\left(\frac{h-q a_{1}}{p}\right)^{q /|p|} .
$$

Then, the variables we use to perform a local study around the point are $\left(a_{1}, u, v\right)$, where $u=\operatorname{Re}(z)$ and $v=\operatorname{Im}(z)$. The expression of the smoothed surface in the new variables is

$$
u^{2}+v^{2}=a_{1}\left(\frac{h-q a_{1}}{p}\right)^{q /|p|} .
$$

Since $u, v$ are not symplectic we modify them to get local symplectic coordinates. We shall achieve it using action-angle coordinates as in Section 5.1, having in mind that we are looking for a $|p|: 1$ covering.

The singularity at $a_{1}=h / q$ is unfolded similarly, but introducing $z$ so that $w=z^{q}$. We get

$$
u^{2}+v^{2}=a_{1}^{|p| / q}\left(\frac{h-q a_{1}}{p}\right),
$$

thus we need to build a $q: 1$ covering, and we shall deal with it by means of adequate action-angle coordinates.

For the singularity $a_{1}=a_{2}=0, h=0$ one has that $p<0$, so $|p|=-p$. Making $w=z^{q+|p|}$, (12) becomes

$$
u^{2}+v^{2}=\left(\frac{q}{|p|}\right)^{q /(q+|p|)} a_{1}
$$

concluding that the symplectic change we shall make is a $(q+|p|): 1$ covering. 
Case $a_{1}=0, h p>0$. This is the smoothing of the peak $P_{2}$. We consider the singular point $\left(a_{1}, a_{3}, a_{4}\right)=$ $(0,0,0)$ when $h \neq 0$, noticing that $a_{2}=h / q \neq 0$. The inequality $|p|>1$ also holds.

Analogously to how we proceeded in the precedent subsection we introduce action-angle coordinates $J_{1}$, $J_{2}, \psi_{1}, \psi_{2}$ from the $I_{k}$ 's, $\theta_{k}$ 's and such that $J_{2}$ depends only on $I_{1}$ and $\psi_{2}$ is a multiple of $p \theta_{1}-q \theta_{2}$. In order to get a symplectic transformation that is a $|p|: 1$ covering we obtain specific values for $J_{k}$ 's, $\psi_{k}$ 's. Next we introduce $J \equiv J_{2}$ and $\psi \equiv \psi_{2}$ where

$$
J=I_{1}, \quad \psi=\theta_{1}-(q / p) \theta_{2} .
$$

The angle $\psi$ is undefined at $a_{1}=0$ but this trouble will be handled below. Note that $\{J, \psi\}=2$.

Putting in (9) $I_{1}, I_{2}$ in terms of $J$ and $h$ (using (26) and the identity $h=q I_{1}+p I_{2}$ ) and writing $\theta_{1}, \theta_{2}$ as functions of $\psi$ the invariants $a_{j}$ 's are given by

$$
\begin{aligned}
& a_{1}=J, \\
& a_{2}=\frac{h-q J}{p}, \\
& a_{3}=J^{|p| / 2}\left(\frac{h-q J}{p}\right)^{q / 2} \cos (p \psi), \\
& a_{4}=-J^{|p| / 2}\left(\frac{h-q J}{p}\right)^{q / 2} \sin (p \psi) .
\end{aligned}
$$

As in the regular case we define $x, y$ in terms of $J$ and $\psi$ through $J=x^{2}+y^{2}, \psi=\tan ^{-1}(y / x)$, thus $\{x, y\}=1$. Using the identities

$$
\begin{aligned}
\left(r^{2}+s^{2}\right)^{|m| / 2} \cos \left[m \tan ^{-1}(s / r)\right] & =\sum_{k=0}^{\lfloor|m| / 2\rfloor}(-1)^{k}\left(\begin{array}{c}
|m| \\
2 k
\end{array}\right) r^{|m|-2 k} s^{2 k} \\
\left(r^{2}+s^{2}\right)^{|m| / 2} \sin \left[m \tan ^{-1}(s / r)\right] & =\operatorname{sgn}(m) \sum_{k=0}^{\lfloor(|m|-1) / 2\rfloor}(-1)^{k}\left(\begin{array}{c}
|m| \\
2 k+1
\end{array}\right) r^{|m|-2 k-1} s^{2 k+1}
\end{aligned}
$$

for reals $r, s$ and integer $m$, we get the unfolding transformation

$$
\begin{aligned}
& a_{1}=x^{2}+y^{2}, \\
& a_{2}=\frac{h-q\left(x^{2}+y^{2}\right)}{p}, \\
& a_{3}=\left[\frac{h-q\left(x^{2}+y^{2}\right)}{p}\right]^{q / 2} \sum_{k=0}^{\lfloor|p| / 2\rfloor}(-1)^{k}\left(\begin{array}{c}
|p| \\
2 k
\end{array}\right) x^{|p|-2 k} y^{2 k}, \\
& a_{4}=-\operatorname{sgn}(p)\left[\frac{h-q\left(x^{2}+y^{2}\right)}{p}\right]^{q / 2} \sum_{k=0}^{\lfloor(|p|-1) / 2\rfloor}(-1)^{k}\left(\begin{array}{c}
|p| \\
2 k+1
\end{array}\right) x^{|p|-2 k-1} y^{2 k+1} .
\end{aligned}
$$

The transformation (29) is well defined near $x=y=0$. In $\left(a_{1}, x, y\right)$-space the transformed surface is given by the smooth constraint $a_{1}=x^{2}+y^{2}$. Since the angle $\psi$ enters in $(27)$ through $\cos (p \psi), \sin (p \psi)$, the change (29) is a $|p|: 1$ covering.

Case $a_{1}=h / q, h>0$. This is the smoothing of the peak $P_{1}$. Now $q>1$ and the singular point is $\left(a_{1}, a_{3}, a_{4}\right)=(h / q, 0,0)$. We make $a_{1}=\left(h-p a_{2}\right) / q$ and introduce $J_{k}$ 's, $\psi_{k}$ 's following the procedure explained in Section 5.1 having in mind that we wish to obtain a $q: 1$ covering. The pair $J \equiv J_{2}, \psi \equiv \psi_{2}$ is

$$
J=I_{2}, \quad \psi=\theta_{2}-(p / q) \theta_{1} .
$$

The angle $\psi$ is not well defined at $a_{2}=0$ but this is not a problem when passing to rectangular coordinates. The action-angle pair satisfies $\{J, \psi\}=2$. 
We replace in (9) $I_{2}$ by $J$ and $I_{1}$ by $(h-p J) / q$ and express $\theta_{1}, \theta_{2}$ in terms of $\psi$ through (30). Then we introduce $x, y$ as in the previous case. With the aid of (28), it is concluded that the $a_{j}$ 's are given by

$$
\begin{aligned}
& a_{1}=\frac{h-p\left(x^{2}+y^{2}\right)}{q}, \\
& a_{2}=x^{2}+y^{2}, \\
& a_{3}=\left[\frac{h-p\left(x^{2}+y^{2}\right)}{q}\right]^{|p| / 2} \sum_{k=0}^{\lfloor q / 2\rfloor}(-1)^{k}\left(\begin{array}{c}
q \\
2 k
\end{array}\right) x^{q-2 k} y^{2 k}, \\
& a_{4}=\left[\frac{h-p\left(x^{2}+y^{2}\right)}{q}\right]^{|p| / 2} \sum_{k=0}^{\lfloor(q-1) / 2\rfloor}(-1)^{k}\left(\begin{array}{c}
q \\
2 k+1
\end{array}\right) x^{q-2 k-1} y^{2 k+1} .
\end{aligned}
$$

The transformation (31) is well defined near $x=y=0$. Transformation (31) is a $q: 1$ covering. In $\left(a_{1}, x, y\right)$ space the transformed surface is given by $a_{1}=\left[h-p\left(x^{2}+y^{2}\right)\right] / q$. The pair $x, y$ is a set of symplectic rectangular coordinates in a neighborhood of $x=y=0$.

Case $a_{1}=0, h=0$. We treat the situation $a_{1}=0$ when $h=0$ so $a_{2}=0$. Observe that $p<0$ and the singular point of $\mathbb{O}$ becomes $(0,0,0)$.

Proceeding analogously to the precedent cases we introduce action-angle coordinates $J_{k}$ 's, $\psi_{k}$ 's assuming that $\psi_{2}$ is a multiple of $p \theta_{1}-q \theta_{2}, J_{2}$ is a function of $I_{1}$ and $I_{2}$ and the change we are constructing is a symplectic $(q+|p|): 1$ covering. Thus we define $J \equiv J_{2}$ and $\psi \equiv \psi_{2}$ by means of

$$
J=I_{1}+I_{2}, \quad \psi=\frac{1}{q+|p|}\left(q \theta_{2}+|p| \theta_{1}\right) .
$$

It is easily checked that $\{J, \psi\}=2$.

The invariants $a_{j}$ 's are written in terms of $J$ and $\psi$. Next rectangular coordinates $x, y$ are introduced as functions of $J$ and $\psi$ as in the previous cases. After some manipulations including making use of (28) we get the unfolding

$$
\begin{aligned}
& a_{1}=\frac{|p|}{q+|p|}\left(x^{2}+y^{2}\right), \\
& a_{2}=\frac{q}{q+|p|}\left(x^{2}+y^{2}\right), \\
& a_{3}=q^{q / 2}|p|^{|p| / 2}(q+|p|)^{(p-q) / 2} \sum_{\substack{k=0 \\
\lfloor(q+|p|) / 2\rfloor}}^{\lfloor(q+|p|-1) / 2\rfloor}(-1)^{k}\left(\begin{array}{c}
q+|p| \\
2 k
\end{array}\right) x^{q+|p|-2 k} y^{2 k}, \\
& a_{4}=q^{q / 2}|p|^{|p| / 2}(q+|p|)^{(p-q) / 2} \sum_{k=0}^{k}\left(\begin{array}{c}
q+|p| \\
2 k+1
\end{array}\right) x^{q+|p|-2 k-1} y^{2 k+1} .
\end{aligned}
$$

The transformation (33) is polynomial, so it is well defined near $x=y=0$. The change $(33)$ is a $(q+|p|): 1$ covering. Since $\{J, \psi\}=2$ then $\{x, y\}=1$.

In $\left(a_{1}, x, y\right)$-space the transformed surface is given by $a_{1}=|p|\left(x^{2}+y^{2}\right) /(q+|p|)$. The variables $x, y$ represent a pair of symplectic rectangular coordinates valid in a neighborhood of $x=y=0$.

In Figure 8 we show the smoothing technique for the $2: 3$ resonance at the points $\left(a_{1}, 0,0\right)=(0,0,0)$ and $\left(a_{1}, 0,0\right)=(h / 2,0,0)$ for the energy level $h=10 / 3$. Around $(0,0,0)$ we employ the transformation (29) and the unfolded surface is drawn in red. For $(h / 2,0,0)$ we use $(31)$ and the unfolded surface is drawn in brown. We also present the smoothing for the 3:-1 resonance at $h=0$ performed through the change (33). The local smoothed surface appears in green. Furthermore we illustrate how a regular point of the trumpet (in dark red) is projected into four points of the surface $a_{1}=\left(x^{2}+y^{2}\right) / 4$, due to the $(q+|p|): 1$ covering.

As in the regular case, given a Hamiltonian in $a$-space when expressing it in $\left(a_{1}, x, y\right)$-space using either (29), (31) or (33) we divide the transformed Hamiltonian by 2.

We summarize the previous paragraphs in the following. 

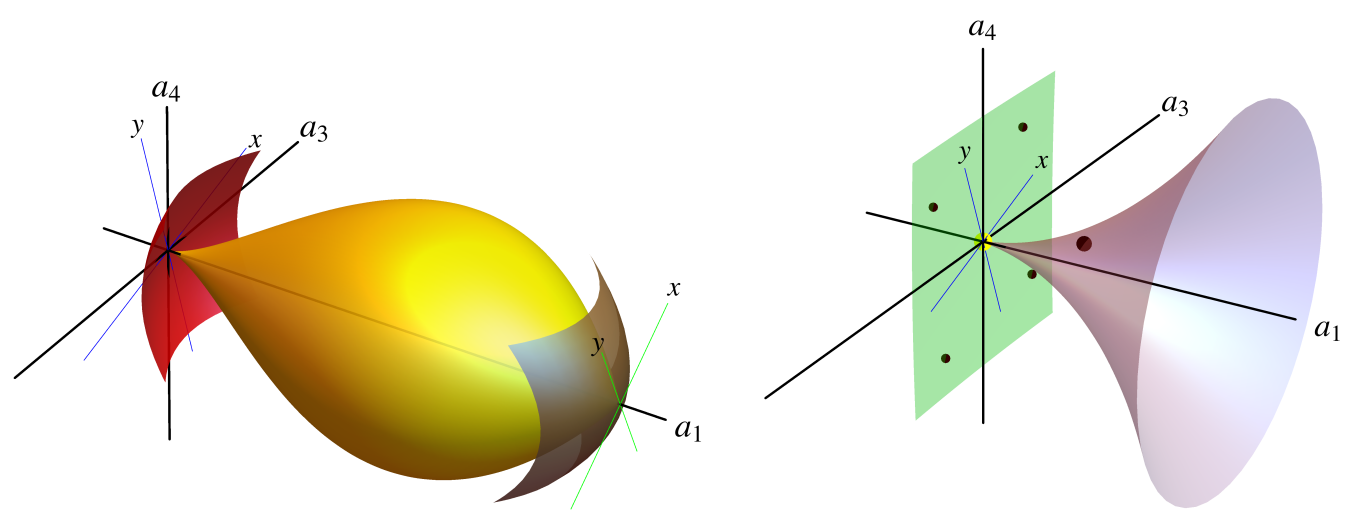

FiguRE 8. Symplectic smoothings for the resonances 2:3 (left) and 3:-1 (right)

Proposition 5.2. Given the Hamiltonian (5) in q:p resonance the surface given through (12) has a singular point of conical type at $\left(a_{1}, a_{3}, a_{4}\right)=(0,0,0)$ when $|p|>1, h p>0$, at $\left(a_{1}, a_{3}, a_{4}\right)=(h / q, 0,0)$ when $q>1$, $h>0$ and at $\left(a_{1}, a_{3}, a_{4}\right)=(0,0,0)$ when $p<0, h=0$. For $|p|>1, h p>0$ an unfolding of the point $(0,0,0)$ is provided by the change (29) together with the constraint $a_{1}=x^{2}+y^{2}$. An unfolding of $(h / q, 0,0)$ for $q>1, h>0$ is provided by (31) with the constraint $a_{1}=\left[h-p\left(x^{2}+y^{2}\right)\right] / q$. When $p<0, h=0$ the unfolding is given by (33) with the constraint $a_{1}=|p|\left(x^{2}+y^{2}\right) /(q+|p|)$.

\section{Attaching Perturbations}

6.1. Normalizing Small Perturbations. Consider a small Hamiltonian perturbation of $\mathcal{H}_{R}$, i.e. take

$$
\mathcal{H}_{\varepsilon}=\mathcal{H}_{R}+\varepsilon \mathcal{H}_{P}
$$

where $\mathcal{H}_{P}$ is a smooth function on $\mathbb{R}^{2 n}$ (the perturbation) and $\varepsilon$ is a small parameter. Our analysis starts with normalizing the Hamiltonian possibly to very high order, i.e. we transform the Hamiltonian by a symplectic change of variables to the form

$$
\mathcal{H}_{\varepsilon}=\mathcal{H}_{R}+\varepsilon \mathcal{H}_{N}+\varepsilon^{2} \mathcal{H}_{H}
$$

where $\mathcal{H}_{N}$ is in normal form with respect to $\mathcal{H}_{R}$, that is, $\left\{\mathcal{H}_{N}, \mathcal{H}_{R}\right\}=0$. Besides it is assumed that $\mathcal{H}_{N}$ is already reduced, which for us means it is written totally in terms of invariants, as a projection $\Pi$ from $\mathcal{N}_{\varepsilon}=\mathcal{H}_{\varepsilon}^{-1}$ to $\mathbb{O}$ has been performed. For the normalization we use the method by Deprit [10]. Since the transformation to normal form does not converge in general it is necessary to stop at a certain order leaving a remainder term $\mathcal{H}_{H}$ called the higher order terms. (In the various applications the powers of $\varepsilon$ will vary.)

6.2. On the Plateau. In order to stress our point of view we recall in this subsection some results about the reconstruction of the flow of a Hamiltonian system related to the points of the reduced space for which regular reduction holds. In particular we give a new description of the center-saddle bifurcation of periodic solutions in the context of regular reduction.

To investigate solutions that are perturbations of plateau points we use averaging as we did in [61]. Specifically let the average of $\mathcal{H}_{P}$ be

$$
\overline{\mathcal{H}}(z)=\frac{1}{2 \pi} \int_{0}^{2 \pi} \mathcal{H}_{P}(Z(t) z) d t .
$$

Clearly $\overline{\mathcal{H}}$ is constant on the orbits of $\mathcal{H}_{R}$ and so, after projecting into $\mathbb{O}$, it can be considered as a function on $\mathbb{O}$ which is smooth on the plateau $L$. (Observe that we keep the same name for the averaged Hamiltonian after projection through $\Pi$.) As a consequence, for Hamiltonians like (35) one has that $\overline{\mathcal{H}}=\mathcal{H}_{N}$, that is, the normal form computation is equivalent to the averaging over the periodic solutions of $\mathcal{H}_{R}$. By Reeb's 
Theorem the plateau has a symplectic structure and $\overline{\mathcal{H}}$ defines a Hamiltonian system on $L$. Let $v$ be symplectic coordinates on $L$ then the Hamiltonian system of equations on $L$ in these coordinates is

$$
\dot{v}=\mathcal{J} \frac{\partial \overline{\mathcal{H}}}{\partial v}
$$

where $\mathcal{J}$ is the usual $(2 n-2) \times(2 n-2)$ matrix of Hamiltonian mechanics.

A critical point $d \in L$ of $\overline{\mathcal{H}}$ (i.e. $\partial \overline{\mathcal{H}} / \partial v(d)=0$ ) is nondegenerate if the Hessian at the critical point, $\partial^{2} \overline{\mathcal{H}} / \partial v^{2}(d)$, is nonsingular. The linearization about the critical point is

$$
\dot{v}=\bar{A} v=\mathcal{J} \frac{\partial^{2} \overline{\mathcal{H}}}{\partial v^{2}}(d) v .
$$

Let the eigenvalues of $\bar{A}$ be $\nu_{1}, \ldots, \nu_{2 n-2}$.

Indeed, all the local results in Section 2 of [61] hold at plateau points. For example:

Theorem 6.1. If $\overline{\mathcal{H}}$ has a nondegenerate critical point at $\Pi(z)=d \in L \subset \mathbb{O}, z \in \mathcal{N}_{\varepsilon}(h)=\mathcal{H}_{\varepsilon}^{-1}(h)$ then there are smooth functions $z(\varepsilon)=z+O(\varepsilon)$ and $T(\varepsilon)=2 \pi+O(\varepsilon)$ for $\varepsilon$ small, such that the solution of $Y_{\varepsilon}$, the vector field associated to $\mathcal{H}_{\varepsilon}$, through $z(\varepsilon)$ is $T(\varepsilon)$-periodic. The characteristic multipliers are

$$
+1,+1,+1+2 \pi \varepsilon \nu_{1}+O\left(\varepsilon^{2}\right), \ldots,+1+2 \pi \varepsilon \nu_{2 n-2}+O\left(\varepsilon^{2}\right) .
$$

Proof. This is an immediate consequence of Corollary 2.2 in [61], where we have used the fact that $T(\varepsilon)=$ $2 \pi+O(\varepsilon)$.

An illustrative bifurcation result is the proof of the existence of an extremal bifurcation of periodic solutions using reduction theory. We could handle other cases such as the pitchfork or the period doubling bifurcations similarly.

For simplicity let $n=2$ so the orbit space $\mathbb{O}$ is 2-dimensional and consider the case where $\mathcal{H}_{P}$ and $\overline{\mathcal{H}}$ depend on a parameter $\delta$ which may be an external parameter or $h \in \mathbb{I} \subset \mathbb{R}$. A critical point $d \in L \subset \mathbb{O}$ of $\overline{\mathcal{H}}$ for $\delta=0$ will be called an extremal critical point $[39,43]$ if there are symplectic coordinates $u, v$ for $\mathbb{O}$ at $d$ such that

$$
\overline{\mathcal{H}}=\overline{\mathcal{H}}_{u}=\overline{\mathcal{H}}_{v}=\overline{\mathcal{H}}_{v v}=\overline{\mathcal{H}}_{u v}=0, \quad \overline{\mathcal{H}}_{u u} \neq 0, \quad \overline{\mathcal{H}}_{v \delta} \neq 0, \quad \overline{\mathcal{H}}_{v v v} \neq 0,
$$

when $u=v=\delta=0$. The canonical example of the extremal critical point is given by

$$
\overline{\mathcal{H}}=u^{2} / 2+\delta v+v^{3} / 3 .
$$

An extremal bifurcation is sometimes called a saddle-center bifurcation, or creation in [1], or fold in catastrophe theory [49].

Lemma 6.1. An extremal critical point $d$ lies on a smooth one-parameter family $d(\delta), d(0)=d$, of critical points of $\overline{\mathcal{H}}$ and $\delta$ achieves a non-degenerate maximum or minimum on this family at $d$. The point $d$ divides the family of critical points in two subfamilies, one subfamily is all saddle points and the other is all non-degenerate maxima (or minima).

This is an elementary result, but for a proof and discussion, see [49].

Let $\mathcal{H}_{\varepsilon, \delta}$ be the Hamiltonian (35) with $\mathcal{H}_{N}=\overline{\mathcal{H}}$, let $Y_{\varepsilon, \delta}$ be the vector field associated to $\mathcal{H}_{\varepsilon, \delta}$ and let $\mathcal{N}_{\varepsilon, \delta}(h)=\mathcal{H}_{\varepsilon, \delta}^{-1}(h)$.

Theorem 6.2. If $\overline{\mathcal{H}}$ has an extremal critical point at $\Pi(z)=d \in L \subset \mathbb{O}$ with $z \in \mathcal{N}_{\varepsilon, 0}(h)$ when $\delta=0$ then there are smooth functions $z(\varepsilon, \delta)$ and $T(\varepsilon, \delta)$ for $\varepsilon$ and $\delta$ small with $z(0,0)=z, T(0,0)=T, z(\varepsilon, \delta) \in \mathcal{N}_{\varepsilon, \delta}(h)$ and the solution of $Y_{\varepsilon, \delta}$ through $z(\varepsilon, \delta)$ is $T(\varepsilon, \delta)$-periodic.

For a fixed small $\varepsilon$ the family of periodic solutions $z(\varepsilon, \cdot)$ has a unique degenerate periodic solution which divides the family into a subfamily of elliptic periodic solutions and a subfamily of hyperbolic periodic solutions.

Proof. We follow the proof found in [15]. From the proof of Theorem 2.2 and Lemma 2.1 of [61] the cross section map in an energy level is of the form $P: \sigma \rightarrow \sigma: y \mapsto P(y, \delta)$ where $y=(u, v)$ are local coordinates in $\mathbb{O}$ and $P(y, \delta)=y+\varepsilon T \mathcal{J} \nabla_{y} \overline{\mathcal{H}}(y, \delta)+O\left(\varepsilon^{2}\right)$. Define $Q$ by $P(y, \delta)=y+\varepsilon T \mathcal{J} Q(y, \delta, \varepsilon)$, 
so $Q(y, \delta, \varepsilon)=\nabla_{y} \overline{\mathcal{H}}(y, \delta)+O(\varepsilon)$. A fixed point of $P$ gives rise to a periodic solution so we must solve $P(y, \delta)=y$ or equivalently $Q(y, \delta, \varepsilon)=0$.

The equations for a fixed point are $Q_{1}(u, v, \delta, \varepsilon)=\overline{\mathcal{H}}_{u}+O(\varepsilon)=0, Q_{2}(u, v, \delta, \varepsilon)=\overline{\mathcal{H}}_{v}+O(\varepsilon)=0$, and when $u=v=\delta=\varepsilon=0$ the Jacobian matrix of these equations is

$$
\frac{\partial\left(Q_{1}, Q_{2}\right)}{\partial(u, v, \delta)}=\frac{\partial\left(\overline{\mathcal{H}}_{u}, \overline{\mathcal{H}}_{v}\right)}{\partial(u, v, \delta)}=\left[\begin{array}{ccc}
\overline{\mathcal{H}}_{u u} & 0 & \overline{\mathcal{H}}_{u \delta} \\
0 & 0 & \overline{\mathcal{H}}_{v \delta}
\end{array}\right]
$$

which is of rank 2 by (37). Thus, we can find functions $\xi(v, \varepsilon)$ and $\eta(v, \varepsilon)$ such that

$$
Q_{1}(\xi(v, \varepsilon), v, \eta(v, \varepsilon), \varepsilon)=Q_{2}(\xi(v, \varepsilon), v, \eta(v, \varepsilon), \varepsilon)=0 .
$$

From these identities we compute

$$
\xi_{v}(0,0)=\eta_{v}(0,0)=0, \quad \eta_{v v}(0,0)=-\overline{\mathcal{H}}_{v v v}(0,0,0) / \overline{\mathcal{H}}_{v \delta}(0,0,0) \neq 0
$$

By the implicit function theorem there is a function $w(\varepsilon)$ such that $\eta_{v}(w(\varepsilon), \varepsilon)=0$ and $\eta_{v v}(w(\varepsilon), \varepsilon) \neq 0$. For a fixed small $\varepsilon$ the function $\eta(v, \varepsilon)$ has a non-degenerate maximum or minimum at $w(\varepsilon)$ and $\eta_{v}(v, \varepsilon)$ changes sign at $w(\varepsilon)$.

Let $E$ be the identity matrix and $A=E+\varepsilon A^{*}$ be a $2 \times 2$ matrix with determinant 1 . From $\operatorname{det} A=1$ obtain that trace $A^{*}=-\varepsilon \operatorname{det} A^{*}$ and so $A$ is elliptic if $\operatorname{det} A^{*}>0$ and it is hyperbolic if $\operatorname{det} A^{*}<0$ for small $\varepsilon$.

Now differentiate the equations $Q_{1}=Q_{2}=0$ with respect to $v$ to get

$$
\begin{aligned}
& Q_{1 u} \xi_{v}+Q_{1 v}+Q_{1 \delta} \eta_{v}=0 \\
& Q_{2 u} \xi_{v}+Q_{2 v}+Q_{2 \delta} \eta_{v}=0,
\end{aligned}
$$

all evaluated at $(\xi(v, \varepsilon), v, \eta(v, \varepsilon), \varepsilon)$. Use Cramer's rule to solve for $\eta_{v}$ to get

$$
\operatorname{det}\left[\begin{array}{ll}
Q_{1 u} & Q_{1 \delta} \\
Q_{2 u} & Q_{2 \delta}
\end{array}\right] \eta_{v}=-\operatorname{det}\left[\begin{array}{ll}
Q_{1 u} & Q_{1 v} \\
Q_{2 u} & Q_{2 v}
\end{array}\right] .
$$

When $\varepsilon=0$ one obtains

$$
\operatorname{det}\left[\begin{array}{ll}
Q_{1 u} & Q_{1 \delta} \\
Q_{2 u} & Q_{2 \delta}
\end{array}\right]=\overline{\mathcal{H}}_{u u}(0,0,0) \overline{\mathcal{H}}_{v \delta} \neq 0
$$

so for small $\varepsilon$ the determinant on the left in (38) is nonzero. Thus,

$$
\operatorname{det}\left[\begin{array}{ll}
Q_{1 u} & Q_{1 v} \\
Q_{2 u} & Q_{2 v}
\end{array}\right]=\operatorname{det}\left[\begin{array}{cc}
Q_{2 u} & Q_{2 v} \\
-Q_{1 u} & -Q_{1 v}
\end{array}\right]
$$

is zero when $v=w(\varepsilon)$, changing sign at this value of $v$.

Using these facts we compute the characteristic multipliers from

$$
\begin{aligned}
\frac{\partial P}{\partial y} & =E+\varepsilon T \mathcal{J} \nabla_{y} Q(\xi(v, \varepsilon), v, \eta(v, \varepsilon), \varepsilon) \\
& =E+\varepsilon T\left[\begin{array}{cc}
Q_{2 u} & Q_{2 v} \\
-Q_{1 u} & -Q_{1 v}
\end{array}\right](\xi(v, \varepsilon), v, \eta(v, \varepsilon), \varepsilon) .
\end{aligned}
$$

Thus for $v=w(\varepsilon)$ the critical point $(\xi(v, \varepsilon), v, \eta(v, \varepsilon))$ is degenerate, a saddle point for $v$ on one side of $w(\varepsilon)$ and a non-degenerate maximum or minimum for $v$ on the other side of $w(\varepsilon)$.

Thus if a reduced Hamiltonian system of two degrees of freedom undergoes an extremal bifurcation then the periodic solutions of the full system do the same. 
6.3. At a Peak. It is possible to approximate the characteristic multipliers of a periodic solution related to a peak. Specifically we have the following result.

Theorem 6.3. Let $P_{s}=d \in \mathbb{O}$ be a peak with frequency $k_{s}$ and $z \in \Pi^{-1}(d)$. Since $k_{j} / k_{s}$ is not an integer for $j \neq s$ then the solution through $z$ of (34) for $\varepsilon=0$ is periodic with period $2 \pi /\left|k_{s}\right|$ and characteristic multipliers

$$
e^{ \pm\left(k_{1} / k_{s}\right) 2 \pi \mathrm{i}}, \ldots, e^{ \pm\left(k_{s} / k_{s}\right) 2 \pi \mathrm{i}}, \ldots, e^{ \pm\left(k_{n} / k_{s}\right) 2 \pi \mathrm{i}} .
$$

Of course $e^{ \pm\left(k_{s} / k_{s}\right) 2 \pi \mathrm{i}}=+1$ as one expects from a periodic solution of a Hamiltonian system, but all the others are not equal to +1 .

For $\varepsilon$ small, the system (34) has a periodic solution near $z$ of period $2 \pi /\left|k_{s}\right|+O(\varepsilon)$ and characteristic multipliers

$$
\begin{aligned}
& e^{ \pm\left(k_{1} / k_{s}\right) 2 \pi \mathrm{i}}+O(\varepsilon), \ldots, e^{ \pm\left(k_{s-1} / k_{s}\right) 2 \pi \mathrm{i}}+O(\varepsilon), \\
& e^{\left(k_{s} / k_{s}\right) 2 \pi \mathrm{i}}=+1, e^{-\left(k_{s} / k_{s}\right) 2 \pi \mathrm{i}}=+1, \\
& e^{ \pm\left(k_{s+1} / k_{s}\right) 2 \pi \mathrm{i}}+O(\varepsilon), \ldots, e^{ \pm\left(k_{n} / k_{s}\right) 2 \pi \mathrm{i}}+O(\varepsilon) .
\end{aligned}
$$

Proof. This result is classical and does not use reduction, see [42, 43].

In order to obtain the $\varepsilon$ order correction terms for the multipliers, decide stability and analyze the bifurcations it is necessary to use symplectic smoothing techniques which are given in Section 5.2 for $n=2$ and are illustrated by examples in Sections 7.2.3 and 7.3.2. The general case when $n \geq 2$ will be discussed in $[46]$.

For $n=2$ we present a result on the characteristic multipliers of a periodic solution related to a peak that improves the approximation given in Theorem 6.3. In this case we have provided symplectic coordinates $v=(x, y)$ given by $(29)$ for the peak $P_{2}$ and by (31) for $P_{1}$ with the aim of making smooth the orbit space around these peaks. Let $\overline{\mathcal{H}}(v)$ be the normal form Hamiltonian written in terms of these coordinates with its associated Hamiltonian equations

$$
\dot{v}=\mathcal{J} \frac{\partial \overline{\mathcal{H}}}{\partial v} .
$$

Since the peak $P_{1}$ is transformed into $(x, y)=(0,0)$ through $(31)$, respectively $P_{1}$ is transformed into $(0,0)$ by means of (29), the linearization about the peak points is

$$
\dot{v}=\bar{A} v=\mathcal{J} \frac{\partial^{2} \overline{\mathcal{H}}}{\partial v^{2}}(0) v .
$$

Let the eigenvalues of $\bar{A}$ be $\pm \nu$.

Theorem 6.4. Let $n=2$ and let $P_{2}=d \in \mathbb{O}$ be a peak with frequency $|p|>1$ and $z \in \Pi^{-1}(d)$. For $\varepsilon$ small the system associated to the Hamiltonian (34), where $\mathcal{H}_{R}$ is given in (5), has a periodic solution near $z$ of period $2 \pi /|p|+O(\varepsilon)$ and characteristic multipliers $+1,+1$ and

$$
e^{(q / p) 2 \pi \mathrm{i}}[1+(2 \pi / p) \varepsilon \nu]+O\left(\varepsilon^{2}\right), e^{-(q / p) 2 \pi \mathrm{i}}[1-(2 \pi / p) \varepsilon \nu]+O\left(\varepsilon^{2}\right) .
$$

For $P_{1}$ simply interchange $p$ and $q$. When $\nu$ is real we assume that it is positive whereas when it is pure imaginary we assume that its imaginary part is positive.

Proof. When $p$ and $q$ are positive refer to the figures in Section 3.2. As in Theorem 6.2 we use the theory of [61], specifically the proof of Theorem 2.2 and Lemma 2.1. Consider the Hamiltonian $\mathcal{H}_{\varepsilon}$ of (34) and compute the cross section map in the energy level $h+O(\varepsilon)$, which is of the form $P: \sigma \rightarrow \sigma: v \mapsto P(v, h)$, where $v=(x, y)$ introduced in (29) are local coordinates in $\mathbb{O}$ valid around the peak point $P_{2}$ and $P(v, h)=$ $v+\varepsilon T \mathcal{J} \nabla_{v} \overline{\mathcal{H}}(v, h)+O\left(\varepsilon^{2}\right)$ and $T=2 \pi /|p|+O(\varepsilon)$. As we know from [61], the fixed point of $P, v=O(\varepsilon)$, leads to the periodic solution related to $P_{2}$.

In case that $P_{2}$ were a critical point in the plateau, the nontrivial characteristic multipliers of the periodic solution would be the eigenvalues of $\partial P(0, h) / \partial v$, where

$$
\frac{\partial P}{\partial v}(0, h)=E+\varepsilon T \mathcal{J} \frac{\partial^{2} \overline{\mathcal{H}}}{\partial v^{2}}(0, h)+O\left(\varepsilon^{2}\right)=E+\varepsilon T \bar{A}+O\left(\varepsilon^{2}\right)
$$


with $\bar{A}$ defined in (40), thus the multipliers would be $1 \pm(2 \pi / p) \varepsilon \nu+O\left(\varepsilon^{2}\right)$. However we need to modify the map $P$ since we have made a $|p|: 1$ covering when introducing coordinates $x, y$ in (29). Thus we have to compose $P$ with a rotating map related to $\mathcal{H}_{q p}$ given in (5). Observe that $x_{1}^{2}+y_{1}^{2}=x^{2}+y^{2}$ then the section map $P^{\prime}$ related to the periodic solution $x=y=0$ (i.e. the periodic solution $P_{2}$ ) is obtained integrating the equations of motion $\dot{x}=\partial \mathcal{H}_{q p} / \partial y=q y, \dot{y}=-\partial \mathcal{H}_{q p} / \partial x=-q x$ between 0 and $2 \pi /|p|$. One gets

$$
v \mapsto P^{\prime}(v)=B v \quad \text { with } \quad B=\left[\begin{array}{cc}
\cos (2 \pi q / p) & \sin (2 \pi q / p) \\
-\sin (2 \pi q / p) & \cos (2 \pi q / p)
\end{array}\right] .
$$

In particular $P^{\prime}$ is the section map corresponding to the cross section $\theta_{2}=0$ associated to the periodic solution $P_{2}$ (the set $S$ in Figure 2).

Composing $P$ with $P^{\prime}$ we get $P^{\prime \prime}=P \circ P^{\prime}$ with $P^{\prime \prime}: \sigma \rightarrow \sigma: v \mapsto P^{\prime \prime}(v, h)=B\left[v+\varepsilon T \mathcal{J} \nabla_{v} \overline{\mathcal{H}}(v, h)\right]+$ $O\left(\varepsilon^{2}\right)$. The Jacobian of $P^{\prime \prime}$ at $v=0$ is

$$
\frac{\partial P^{\prime \prime}}{\partial v}(0, h)=B+\varepsilon T B \bar{A}+O\left(\varepsilon^{2}\right)
$$

and its eigenvalues are the approximate characteristic multipliers of the periodic solution related to $P_{2}$. These eigenvalues are given in (41).

The reader could notice that the factor $1 / p$ in the multipliers (41) comes from the fact that we have performed a $|p|: 1$ covering when passing to the coordinates $x, y$.

6.4. At a Ridge. Ridges can only be found in systems with three or more degrees of freedom and one is not assured that a periodic solution is generated from a ridge in general. We will develop the general theory of symplectic smoothing for ridges in [46].

\section{Bifurcations in the $q: p$ Resonance}

7.1. Cherry's Example: 2:-1 Resonance. To show that linear stability does not imply nonlinear stability for Hamiltonian systems Whittaker [60] gives an example that he attributes to Cherry [5]. In his original variables the differential equation is

$$
\frac{d x_{1}}{d t}=\frac{\partial H}{\partial y_{1}}, \quad \frac{d x_{2}}{d t}=\frac{\partial H}{\partial y_{2}}, \quad \frac{d y_{1}}{d t}=-\frac{\partial H}{\partial x_{1}}, \quad \frac{d y_{2}}{d t}=-\frac{\partial H}{\partial x_{2}},
$$

where

$$
H=\frac{1}{2} \lambda\left(x_{1}^{2}+y_{1}^{2}\right)-\lambda\left(x_{2}^{2}+y_{2}^{2}\right)+\frac{1}{2} \alpha\left[x_{2}\left(x_{1}^{2}-y_{1}^{2}\right)-2 x_{1} y_{1} y_{2}\right],
$$

with $\lambda$ and $\alpha$ arbitrary parameters. He explicitly gives solutions

$$
\begin{array}{ll}
x_{1}=\frac{\sqrt{2}}{\alpha(t+\epsilon)} \sin (\lambda t+\gamma), & y_{1}=\frac{\sqrt{2}}{\alpha(t+\epsilon)} \cos (\lambda t+\gamma), \\
x_{2}=\frac{1}{\alpha(t+\epsilon)} \sin [2(\lambda t+\gamma)], & y_{2}=\frac{-1}{\alpha(t+\epsilon)} \cos [2(\lambda t+\gamma)],
\end{array}
$$

where $\epsilon$ and $\gamma$ are constants of integration. These solutions represent orbits which spiral to the origin as $t \rightarrow+\infty$ and $t \rightarrow-\infty$, but which have branches with all coordinates spiraling to infinity as $t$ tends to $-\epsilon$. Think of these solutions on two surfaces in $\mathbb{R}^{4}$. The equilibrium at the origin is therefore unstable, in spite of being stable to the first order.

Whittaker gives no hint as to the origin of this example - no discussion of normal forms or invariants. Cherry on the other hand was looking at systems which have an additional integral and hence were solvable. A closer look at this example reveals that the system is in 2:-1 resonance and the Hamiltonian is written in terms of our invariants. To be consistent with our notation we specialize by taking $\lambda=-1, \alpha=1$ and reverse the subscripts so that

$$
H=2 a_{1}-a_{2}+a_{3} .
$$

The orbit space $\mathbb{O}$ when $2 a_{1}-a_{2}=h$ is the surface

$$
a_{3}^{2}+a_{4}^{2}=a_{1}\left(h-2 a_{1}\right)^{2}, \quad a_{1} \geq 0, \quad a_{1} \geq h / 2 .
$$


The Hamiltonian $H$ is already in normal form and so the reduced Hamiltonian is $\bar{H}=a_{3}$. Using the Poisson structure given in Table 1 with $q=2$ and $p=-1$, we obtain the equations of motion, arriving at

$$
\dot{a}_{1}=\left\{a_{1}, \bar{H}\right\}=-2 a_{4}, \quad \dot{a}_{3}=\left\{a_{3}, \bar{H}\right\}=0, \quad \dot{a}_{4}=\left\{a_{4}, \bar{H}\right\}=-a_{2}\left(4 a_{1}+a_{2}\right) .
$$

The system has also been studied in [45] using the invariants $a_{k}$ 's. Recall that on the orbit space $a_{2}=2 a_{1}-h$. For a critical point one must have $a_{4}=0$ and $a_{2}\left(4 a_{1}+a_{2}\right)=0$ and since not both $a_{1}$ and $a_{2}$ can be zero or negative the conditions for an equilibrium are $a_{2}=a_{4}=0$. But in the orbit space $a_{2}=0$ only when $a_{1}=h / 2$ and that occurs when $h \geq 0$; this is a singular point of the orbit space (a peak when $h>0$ ) which is always an equilibrium of the reduced system even when the equations of motion are not well defined in non-regular points. When $h \geq 0$ the orbit space is a trumpet whereas for $h<0$ it is a cap.

Look at the flow lines in Figure 9. The flow lines lie in $\bar{H}=a_{3}=$ constant and $a_{4}$ is decreasing. These flow lines can be interpreted as approximations of the flow of the two degrees of freedom problem in symplectic charts on the orbit space. These charts are the different trumpets and caps defined when varying $h$.

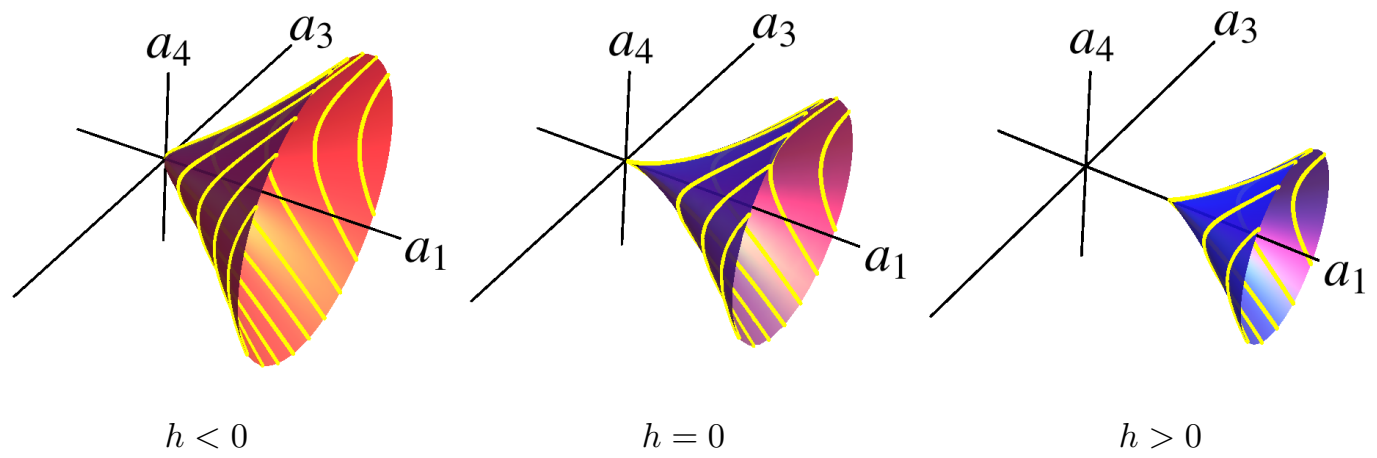

Figure 9. Flow of Cherry's example in $\mathbb{O}$

When $h=0$ the origin in $a$-space corresponds to the origin in $\mathbb{R}^{4}$ and it is a singularity (not a peak). There is an orbit on $\mathbb{O}$ tending to the origin as $t \rightarrow+\infty$ and there is an orbit on $\mathbb{O}$ tending to the origin as $t \rightarrow-\infty$. These represent a surface of solutions that spiral to the origin as $t \rightarrow \pm \infty$ and are geometrically the solutions given by Whittaker in (42).

When $h>0$ there is an equilibrium (a peak) on $\mathbb{O}$ at $a_{1}=h / 2, a_{3}=a_{4}=0$. This gives rise to a periodic solution of period $T \sim \pi$ for each $h \geq 0$. These solutions form the short periodic family given by Liapunov Center Theorem [43]. Note that here too there is an orbit on $\mathbb{O}$ tending to the equilibrium as $t \rightarrow \pm \infty$. Thus the solutions in the short period family are unstable.

When $h<0$ there are no equilibria and so all solutions recede far away as $t \rightarrow \pm \infty$.

7.2. Detuning the 2:-1 Resonance. Look at a detuning of the previous example, namely,

$$
H=(2+\mu) a_{1}-a_{2}+a_{3},
$$

where $\mu$ is a parameter. This system has also been dealt with partially in [55] and in [9]. We also treated it in the setting of singular reduction in [45]. Here we give a much deeper insight using the results of Sections 5 and 6 . In particular the bifurcation analysis by means of the symplectic smoothing is new.

When $2 a_{1}-a_{2}=h$ the orbit space is still the surface

$$
a_{3}^{2}+a_{4}^{2}=a_{1}\left(h-2 a_{1}\right)^{2}, \quad a_{1} \geq 0, \quad a_{1} \geq h / 2 .
$$

7.2.1. Equations of Motion and Equilibria. The reduced Hamiltonian on the orbit space is $\bar{H}=\mu a_{1}+a_{3}$ and the equations of motion are obtained through the Poisson brackets of Table 1, yielding that

$$
\dot{a}_{1}=\left\{a_{1}, \bar{H}\right\}=-2 a_{4}, \quad \dot{a}_{3}=\left\{a_{3}, \bar{H}\right\}=2 \mu a_{4}, \quad \dot{a}_{4}=\left\{a_{4}, \bar{H}\right\}=-2 \mu a_{3}-a_{2}\left(4 a_{1}+a_{2}\right) .
$$

Recall that on the orbit space $a_{2}=2 a_{1}-h$. For an equilibrium point one must have

$$
a_{4}=0, \quad-2 \mu a_{3}-a_{2}\left(4 a_{1}+a_{2}\right)=0, \quad a_{2}=2 a_{1}-h, \quad a_{3}^{2}+a_{4}^{2}=a_{1}\left(h-2 a_{1}\right)^{2},
$$


with $a_{1} \geq \max \{0, h / 2\}$. Solving (44) in $\left\{a_{1}, a_{3}, a_{4}\right\}$ we obtain up to three solutions, namely,

$$
\begin{aligned}
P_{1} & =(h / 2,0,0), \\
L_{ \pm} & =\left(\frac{1}{18}\left(3 h+\mu^{2} \pm|\mu| \sqrt{6 h+\mu^{2}}\right), \frac{1}{27 \mu}\left[-\mu^{4} \pm|\mu|\left(3 h-\mu^{2}\right) \sqrt{6 h+\mu^{2}}\right], 0\right) .
\end{aligned}
$$

These solutions correspond to critical points of the system (44) when the three components of each point are real numbers and $a_{1} \geq \max \{0, h / 2\}$. Indeed the point $P_{1}$ should be discarded from the solutions of (44) as the orbit space $\mathbb{O}$ is not defined in it since it is a singular point. However it is always an equilibrium of a reduced system defined in $\mathbb{O}$. We get

(i) $P_{1}$ is an equilibrium for $h \geq 0$, that is, in regions I, II of Figure 10 .

(ii) $L_{+}$is an equilibrium when $-\mu^{2} / 6 \leq h \leq \mu^{2} / 2$, i.e. in regions II and III of Figure 10.

(iii) $L_{-}$is an equilibrium when $-\mu^{2} / 6 \leq h \leq 0$, i.e. in region III of Figure 10.

The value of $h$ as a function of the Hamiltonian $H=e$ on the three equilibria is represented in Figure 11. The flow of the reduced system appears in Figure 12.

7.2.2. Bifurcation Curves. Bifurcations of equilibria occur when a critical point appears or disappears or when two or the three possible equilibria collide. Solving the equations for this to happen we end up with the bifurcation plane appearing in Figure 10. The plane is symmetric with respect to $\mu=0$, so we only plot the bifurcation lines for $\mu \geq 0$.

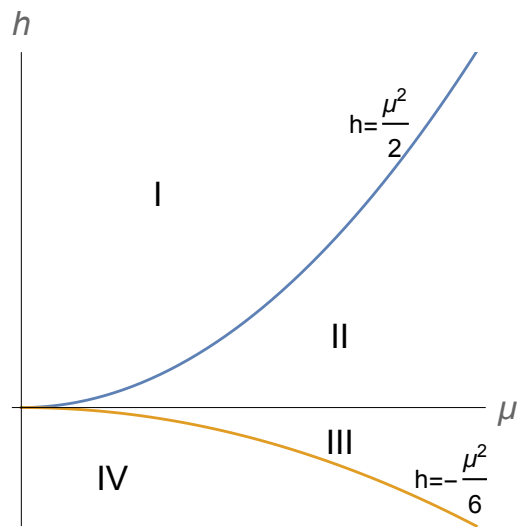

FiguRE 10. Bifurcation plane in the detuning of the 2:-1 resonance

In region I, when $h \geq \mu^{2} / 2$, the orbit space has a peak at $P_{1}$ and it is the only equilibrium point.

In region II the orbit space $\mathbb{O}$ has a peak at $P_{1}$ and the point $L_{+}$is also an equilibrium in the plateau. When $h \lesssim \mu^{2} / 2$ the point $L_{+}$approaches $P_{1}$ and finally collides with it when $h=\mu^{2} / 2$. See the point where the green and the red lines meet in Figure 11.

For $h=0$ the situation is qualitatively the same as in region II, but the singularity moves to the origin of $a$-space.

For $h<0$ the orbit space becomes regular and $P_{1}$ is not an equilibrium. The point $L_{-}$appears as an equilibrium up to $h=-\mu^{2} / 6$ while $L_{+}$still persists for these values. This situation corresponds to region III of Figure 10. For values of $h \lesssim 0$ the point $L_{-}$, which is in the plateau, is close to $P_{1}$ colliding with it when $h=0$, and only the point $P_{1}$ survives for $h>0$ as a peak. See the point where the red and the blue lines meet in Figure 11.

When $h \gtrsim-\mu^{2} / 6$ the two equilibria $L_{+}$and $L_{-}$get close until they collide when $h=-\mu^{2} / 6$. After crossing this line, once in region IV, the equilibrium disappears, i.e., for $h<-\mu^{2} / 6$ there are no equilibria. See Figure 12 and the point where the blue and the green lines meet in Figure 11.

When $h=\mu=0$ the only equilibrium is the origin of the orbit space. 


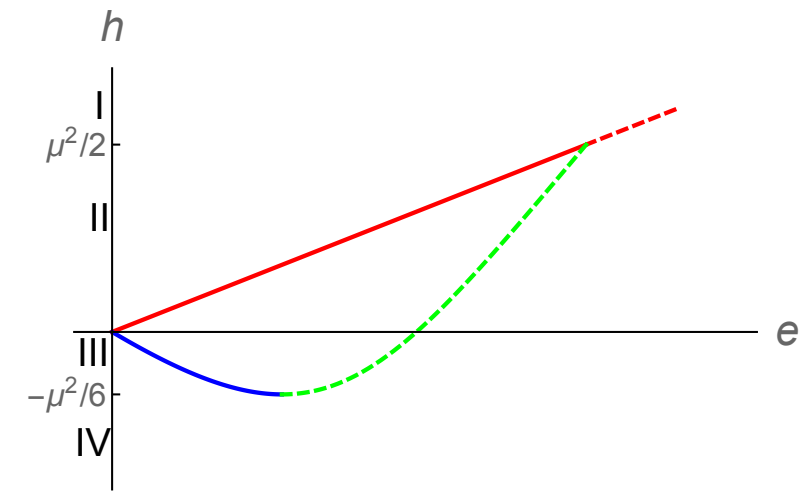

Figure 11. Value of $h$ at each equilibrium as a function of $H=e$ for $\mu$ fixed. Solid lines correspond to elliptic points and dashed lines to hyperbolic ones. The red line is associated to $P_{1}$, the blue line to $L_{-}$and the green one to $L_{+}$. Bifurcations take place where two different colors meet, in particular a center-saddle bifurcation occurs at $h=-\mu^{2} / 6$; a vanishing of periodic solution at $h=0$; and a subcritical Hamiltonian flip bifurcation when $h=\mu^{2} / 2$
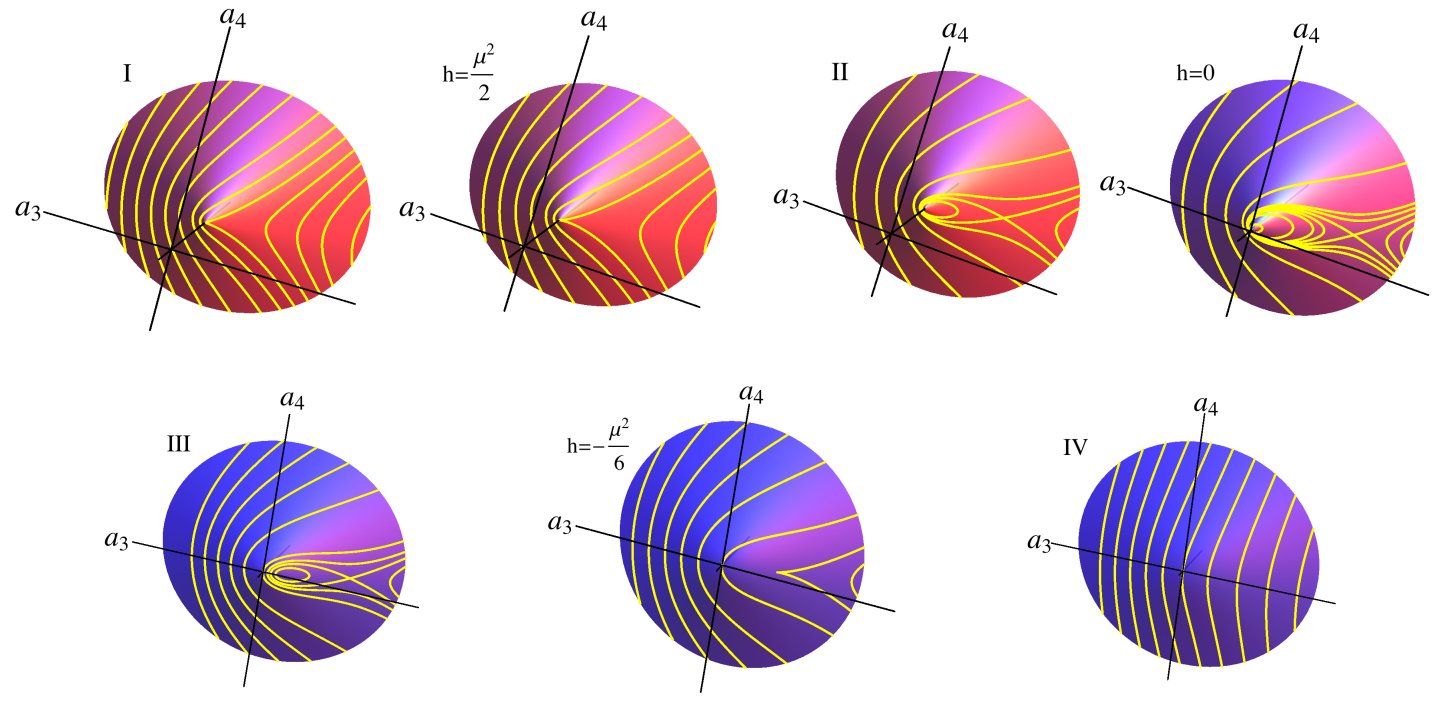

FiguRE 12. Flow in the different regions of the bifurcation plane for the detuning of the 2:-1 resonance

7.2.3. Bifurcation Analysis and Reconstruction. In order to determine the stability of the equilibria and classify their bifurcations we distinguish between singularities and points in the plateau.

Hamiltonian flip bifurcation: The peak, $P_{1}$, is associated to a $\pi$-periodic solution. Its stability is analyzed applying the symplectic smoothing technique of Section 5.2, defining a set of adequate coordinates around $P_{1}$. We use the transformation (31) with $q=2$ and $p=-1$, obtaining

$$
a_{1}=\frac{1}{2}\left(h+x^{2}+y^{2}\right), a_{2}=x^{2}+y^{2}, a_{3}=\frac{1}{\sqrt{2}}\left(x^{2}-y^{2}\right) \sqrt{h+x^{2}+y^{2}}, a_{4}=\sqrt{2} x y \sqrt{h+x^{2}+y^{2}} .
$$

The change (45) is applied to $\bar{H} / 2$ and making a Taylor expansion around $(0,0)$ one ends up with

$$
\bar{H}=\frac{\sqrt{h}}{2 \sqrt{2}}\left(x^{2}-y^{2}\right)+\frac{\mu}{4}\left(x^{2}+y^{2}\right)+\frac{1}{4 \sqrt{2 h}}\left(x^{4}-y^{4}\right)+\ldots,
$$


after discarding constant terms. The ellipsis stands for terms that are at least sixth order. The expansion is valid for $h>0$.

Hamiltonian $\bar{H}$ represents a $\mathbb{Z}_{2}$-symmetric one degree of freedom system in $\left(a_{1}, x, y\right)$-space. The eigenvalues of the Hessian matrix related to $\bar{H}$ evaluated at $(x, y)=(0,0)$ (i.e., the point corresponding to the peak in $a$-space) are $\nu_{ \pm}= \pm(1 / 2) \sqrt{2 h-\mu^{2}}$, thus $(0,0)$ is unstable (a saddle) for $h>\mu^{2} / 2$ and stable (a center) for $0<h<\mu^{2} / 2$. For $h=\mu^{2} / 2$ it is a cusp point and the normal form around it is $x^{2}-y^{4} /\left(2 \mu^{2}\right)$ for $\mu>0$, hence it is unstable. Note that the stability of the peak is difficult to guess from Figure 12, as the flow in region I and on the bifurcation $h=\mu^{2} / 2$ look alike. Calculating the other valid critical points of the system in $\left(a_{1}, x, y\right)$-space from the 4-jet above we see that for $h>\mu^{2} / 2$ the origin is the only equilibrium, whereas for $0<h<\mu^{2} / 2$ two more hyperbolic equilibria (saddles) arise. This is the typical scenario of a subcritical Hamiltonian pitchfork bifurcation occurring at $h=\mu^{2} / 2$. The coordinates $(x, y)$ of the two saddles are $\left(0, \pm(h / 2)^{1 / 4} \sqrt{\mu-(2 h)^{1 / 2}}\right)$ and their eigenvalues $\pm(1 / \sqrt{2}) \sqrt{\mu^{2}-2 h}$. Returning to $a$-space the previous considerations tell us that the reduced Hamiltonian $\bar{H}$ undergoes a subcritical Hamiltonian flip (or period doubling) bifurcation in $\mathbb{O}$ because the two hyperbolic equilibria become a unique saddle, this point being $L_{+}$; see Figure 12 .

Reconstructing the dynamics of the full system in $\mathbb{R}^{4}$ we conclude that there is a Hamiltonian flip bifurcation of periodic solutions. The unstable periodic solution corresponding to the peak point has period near $\pi$ and bifurcates at $h \approx \mu^{2} / 2$, changing its stability character and giving rise to an unstable periodic solution whose period is near $2 \pi$. Applying Theorem 6.4, the approximate non-trivial multipliers of the periodic solution related to $P_{1}$ are $e^{(-1 / 2) 2 \pi \mathrm{i}}\left[1+(2 \pi / 2) \nu_{+}\right]$and $e^{-(-1 / 2) 2 \pi \mathrm{i}}\left[1+(2 \pi / 2) \nu_{-}\right]$, that is, $-1 \pm(\pi / 2) \sqrt{2 h-\mu^{2}}$. We exclude the case $\mu=0$ in the analysis.

Vanishing of the periodic solution: We study the behavior of $H$ when $h$ passes through 0 . When $h \gtrsim 0$ the peak is stable while for $h \lesssim 0$ it becomes a regular non-critical point and near to it the point $L_{-}$emerges as a regular point. For $h=0$ the stability of the singular point corresponds to the stability of the origin in $\mathbb{R}^{4}$ for $H$ in the coordinates $\left(x_{1}, y_{1}, x_{2}, y_{2}\right)$ and we analyze it by applying the change (33). The unfolding is given by

$$
a_{1}=\frac{1}{3}\left(x^{2}+y^{2}\right), \quad a_{2}=\frac{2}{3}\left(x^{2}+y^{2}\right), \quad a_{3}=\frac{2}{3 \sqrt{3}} x\left(x^{2}-3 y^{2}\right), \quad a_{4}=\frac{2}{3 \sqrt{3}} y\left(3 x^{2}-y^{2}\right) .
$$

The reduced Hamiltonian reads as

$$
\bar{H}=\frac{1}{6} \mu\left(x^{2}+y^{2}\right)+\frac{1}{3 \sqrt{3}} x\left(x^{2}-3 y^{2}\right),
$$

which is a $\mathbb{Z}_{3}$-symmetric Hamiltonian. We get that the origin is an elliptic point whenever $\mu \neq 0$ whereas for $h=\mu=0$ it is a degenerate unstable point. Concretely the eigenvalues of $(0,0)$ are $\pm \mu \mathrm{i} / 3$. Apart from the origin we obtain three saddle points with coordinates $(-\mu / \sqrt{3}, 0),(\mu /(2 \sqrt{3}), \pm \mu / 2)$ and eigenvalues $\pm \mu / \sqrt{3}$, provided $\mu \neq 0$. The three saddles correspond to the point $L_{+}$in the orbit space $\mathbb{O}$. See Figure 13 where the $3: 1$ covering induced by the transformation (46) is clearly discerned.

Dealing with the flow of $H$ in $\mathbb{R}^{4}$ we know that when $h \gtrsim 0$ the point $P_{1}$ represents a stable periodic solution with period close to $\pi$. For $h \lesssim 0$ the singularity disappears but $L_{-}$is born close to the point $(0,0,0)$ in $a$-space as a regular point. Thus $L_{-}$corresponds to a periodic solution of the full system with period near $2 \pi$. Hence the stable periodic solution of period near $\pi$ shrinks down to the origin when $h=0$ and reappears for $h<0$ also as a stable periodic solution though duplicating its period.

Center-saddle bifurcation: In this case the bifurcation occurs in the plateau of $\mathbb{O}$ thus we apply the theory of Section 6.2. We also make use of the local transformation (18). Let $\left(a_{1}^{0}, a_{3}^{0}, a_{4}^{0}\right)$ represent either the coordinates of $L_{+}$or of $L_{-}$. Applying (21) we obtain $\left(x_{0}, y_{0}\right)=\left(-\frac{1}{6}\left(\mu \pm \sqrt{6 h+\mu^{2}}\right), 0\right)$ with the upper sign for $L_{+}$and the lower one for $L_{-}$. Thus we define symplectic coordinates $\bar{x}, \bar{y}$ according to the study carried out in Section 5.1. By means of (18) we get

$$
a_{1}=x^{2}+y^{2}, \quad a_{2}=2\left(x^{2}+y^{2}\right)-h, \quad a_{3}=x\left[2\left(x^{2}+y^{2}\right)-h\right], \quad a_{4}=y\left[2\left(x^{2}+y^{2}\right)-h\right],
$$



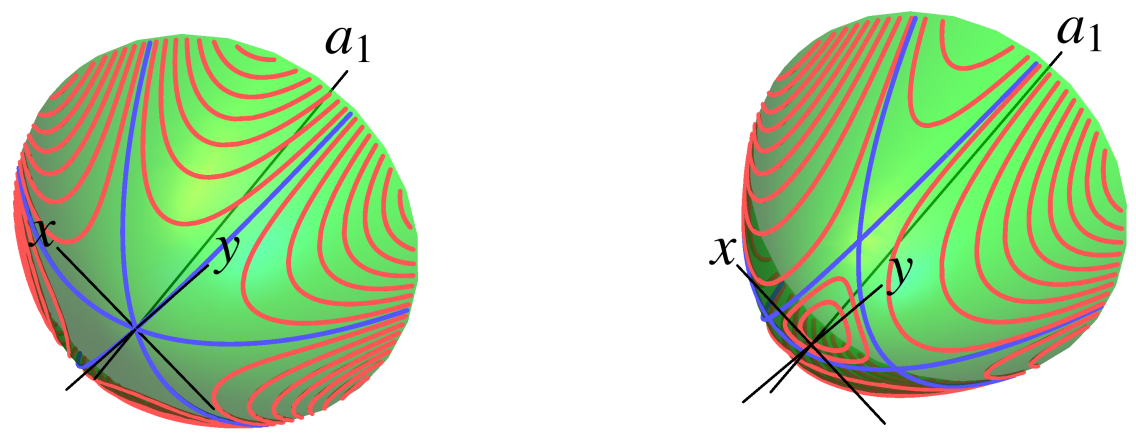

Figure 13. Symplectic smoothing of the $2:-1$ resonance for $h=0$ and $\mu=0$ (left) and for $h=0$ and $\mu=2$ (right)

and replace $x, y$ by $\bar{x}+x_{0}, \bar{y}+y_{0}$ where $x_{0}, y_{0}$ are taken differently for $L_{+}$or for $L_{-}$. Plugging the changes in $\bar{H}$, dividing the result by two, expanding in terms of $\bar{x}, \bar{y}$ and dropping constant terms we end up with

$$
\bar{H}=\frac{1}{3} \mu \bar{y}^{2} \mp \frac{1}{6} \sqrt{6 h+\mu^{2}}\left(3 \bar{x}^{2}+\bar{y}^{2}\right)+\bar{x}\left(\bar{x}^{2}+\bar{y}^{2}\right)+\ldots,
$$

where the upper sign is for $L_{+}$and the lower one for $L_{-}$and the ellipsis means that the remaining terms start at degree four. The eigenvalues are

$$
\pm \frac{1}{\sqrt{3}} \sqrt{-6 h-\mu\left(\mu-2 \sqrt{6 h+\mu^{2}}\right)} \text { for } L_{+}, \quad \pm \frac{1}{\sqrt{3}} \sqrt{-6 h-\mu\left(\mu+2 \sqrt{6 h+\mu^{2}}\right)} \text { for } L_{-} .
$$

We conclude that $L_{-}$is an elliptic equilibrium when $-\mu^{2} / 6<h<0$, i.e. in region III, see Figures 11 and 12 , whereas $L_{+}$remains a saddle when $-\mu^{2} / 6 \lesssim h$. For $h=-\mu^{2} / 6$ there is a collision between $L_{+}$and $L_{-}$that gives rise to an extremal point that disappears once in region IV, see Figure 12 . We can use the 3-jet given above either in a neighborhood of $L_{+}$or of $L_{-}$to check that the normal form of the bifurcation happening at $h=-\mu^{2} / 6$ corresponds to the one studied in Section 6.2. In summary, at $h=-\mu^{2} / 6$ there is a center-saddle bifurcation of relative equilibria in the orbifold $\mathbb{O}$.

Applying Theorem 6.1 we assure the existence of two (families of) periodic solutions for the Hamiltonian $H$ related to $L_{+}$and $L_{-}$whose periods are near $2 \pi$. Besides the periodic solution associated to $L_{-}$is elliptic while the one associated to $L_{+}$is hyperbolic and their non-trivial approximate multipliers are, respectively, $1 \pm(2 / \sqrt{3}) \pi\left[-6 h-\mu\left(\mu+2 \sqrt{6 h+\mu^{2}}\right)\right]^{1 / 2}$ and $1 \pm(2 / \sqrt{3}) \pi\left[-6 h-\mu\left(\mu-2 \sqrt{6 h+\mu^{2}}\right)\right]^{1 / 2}$. These multipliers are valid whenever the periodic solutions exist. According to Theorem 6.2 , these periodic solutions undergo a center-saddle bifurcation in $\mathbb{R}^{4}$ when $h \approx-\mu^{2} / 6$.

\subsection{The 3:2 Resonance.}

7.3.1. Hamiltonian, Equations of Motion and Relative Equilibria. We consider the detuning of the 3:2 resonance as it is presented and analyzed by Schmidt in [55]. The starting point is the Hamiltonian

$$
H=3 a_{1}+2 a_{2}+\varepsilon^{2}\left(\lambda_{1} a_{1}+\lambda_{2} a_{2}+\frac{A}{2} a_{1}^{2}+B a_{1} a_{2}+\frac{C}{2} a_{2}^{2}\right)+\varepsilon^{3}\left(D_{1} a_{3}+D_{2} a_{4}\right)
$$

with the relations

$$
a_{2}=\frac{1}{2}\left(h-3 a_{1}\right), \quad a_{3}^{2}+a_{4}^{2}=\frac{1}{8} a_{1}^{2}\left(h-3 a_{1}\right)^{3}, \quad a_{1} \geq 0, \quad a_{2} \geq 0
$$

where we have fixed an energy level $h>0$. The constants $A, B, C, D_{1}, D_{2}$ have real values, $\lambda_{1}$ and $\lambda_{2}$ are detuning parameters and $\varepsilon$ is a small parameter. The orbit space is the second constraint of (48) together with $0 \leq a_{1} \leq h / 3$. The reduced Hamiltonian is given by

$$
\bar{H}=\lambda_{1} a_{1}+\lambda_{2} a_{2}+\frac{A}{2} a_{1}^{2}+B a_{1} a_{2}+\frac{C}{2} a_{2}^{2}+\varepsilon\left(D_{1} a_{3}+D_{2} a_{4}\right) .
$$


Apart from the peaks, the relative equilibria are the solutions of the system

$$
\begin{aligned}
& \dot{a}_{1}=\left\{a_{1}, \bar{H}\right\}=4 \varepsilon\left(a_{4} D_{1}-a_{3} D_{2}\right)=0, \\
& \dot{a}_{3}=\left\{a_{3}, \bar{H}\right\}=-\left[h(2 B-3 C)+2\left(2 \lambda_{1}-3 \lambda_{2}\right)\right] a_{4}-(4 A-12 B+9 C) a_{1} a_{4} \\
& +\frac{\varepsilon}{4} D_{2}\left(15 a_{1}-2 h\right)\left(h-3 a_{1}\right)^{2} a_{1}=0, \\
& \dot{a}_{4}=\left\{a_{4}, \bar{H}\right\}=\left[h(2 B-3 C)+2\left(2 \lambda_{1}-3 \lambda_{2}\right)\right] a_{3}+(4 A-12 B+9 C) a_{1} a_{3} \\
& -\frac{\varepsilon}{4} D_{1}\left(15 a_{1}-2 h\right)\left(h-3 a_{1}\right)^{2} a_{1}=0, \\
& a_{3}^{2}+a_{4}^{2}=\frac{1}{8} a_{1}^{2}\left(h-3 a_{1}\right)^{3} .
\end{aligned}
$$

From the first equation we infer that the solutions $\left(a_{1}, a_{3}, a_{4}\right)$ satisfy $a_{4} D_{1}=a_{3} D_{2}$. Then, considering the generic case $D_{1}, D_{2} \neq 0$ and substituting this relation in the second and third equations we arrive at $\dot{a}_{3} D_{1}=-\dot{a}_{4} D_{2}$. Therefore our system can be reduced to two equations in $\left(a_{1}, a_{3}\right)$, which are the third and the fourth ones. From the third equation it is natural to define the following parameters, as it is done in $[55]$,

$$
M=2 B-3 C, \quad \sigma=2 \lambda_{1}-3 \lambda_{2}, \quad \Delta=4 A-12 B+9 C .
$$

The relevant factors of the resultant of these two equations with respect to $a_{3}$ are

$$
\begin{aligned}
\mathcal{R}\left(a_{1}\right)=a_{1}^{2}\left(3 a_{1}-h\right)^{3} & \left\{675 \varepsilon^{2}\left(D_{1}^{2}+D_{2}^{2}\right) a_{1}^{3}+\left[2 \Delta^{2}-405 h \varepsilon^{2}\left(D_{1}^{2}+D_{2}^{2}\right)\right] a_{1}^{2}\right. \\
+ & 4\left[18 h^{2} \varepsilon^{2}\left(D_{1}^{2}+D_{2}^{2}\right)+\Delta(h M+2 \sigma)\right] a_{1} \\
& \left.+2\left[-2 h^{3} \varepsilon^{2}\left(D_{1}^{2}+D_{2}^{2}\right)+(h M+2 \sigma)^{2}\right]\right\} .
\end{aligned}
$$

The roots $a_{1}=0, h / 3$ lead to the peak points in $\mathbb{O}$. (Strictly speaking one cannot conclude the existence of the peaks from the equations of motion, however they are critical points of the reduced system.) The peak $P_{1}=(h / 3,0,0)$ corresponds to the $q=3$ singularity in $\mathbb{O}$, i.e. the family of short periodic solutions in $\mathbb{R}^{4}$. The peak $P_{2}=(0,0,0)$ corresponds to the $p=2$ singularity in $\mathbb{O}$, i.e. the family of long period solutions in $\mathbb{R}^{4}$. The solutions of the equation $\mathcal{R}\left(a_{1}\right)=0$ with $0<a_{1}<h / 3$ are related to the critical points in the plateau $L \subset \mathbb{O}$. So they are the roots of the cubic polynomial in $a_{1}$ given by

$$
\begin{aligned}
p_{3}\left(a_{1}\right)= & 675 D a_{1}^{3}+\left(2 \Delta^{2}-405 h D\right) a_{1}^{2}+4\left[18 h^{2} D+\Delta(h M+2 \sigma)\right] a_{1} \\
& +2\left[(h M+2 \sigma)^{2}-2 h^{3} D\right],
\end{aligned}
$$

such that $0<a_{1}<h / 3$. We have introduced the new parameter $D=\varepsilon^{2}\left(D_{1}^{2}+D_{2}^{2}\right)$.

The bifurcations take place when there is a multiple root of the cubic polynomial, say $a_{1}^{0}$, such that $0<a_{1}^{0}<h / 3$ or when a root of this cubic polynomial is 0 or $h / 3$.

(i) The appearance of multiple valid roots of the cubic polynomial corresponds to a multiple collision of relative equilibria in the plateau. This occurs when the discriminant $\mathcal{D}$ of the cubic polynomial is zero, where $\mathcal{D}$ is

$$
\begin{aligned}
\mathcal{D}=4 D[2 \Delta h+15(h M+2 \sigma)]^{2}\{ & 8 \Delta^{4} h+24 \Delta^{3}(h M+2 \sigma)-3564 \Delta^{2} h^{2} D \\
& -29160 \Delta h D(h M+2 \sigma) \\
& \left.+10935 D\left[-5(h M+2 \sigma)^{2}+2 h^{3} D\right]\right\} .
\end{aligned}
$$

So when $\mathcal{D}=0$ the cubic polynomial has a multiple root, but we need $0<a_{1}^{0}<h / 3$ and it is not possible when $2 \Delta h+15(h M+2 \sigma)=0$, but only when the last factor of $\mathcal{D}$ vanishes. Thence a bifurcation in the plateau takes place when

$$
\begin{aligned}
\Gamma_{1} \equiv & 8 \Delta^{4} h+24 \Delta^{3}(h M+2 \sigma)-3564 \Delta^{2} h^{2} D-29160 \Delta h D(h M+2 \sigma) \\
& +10935 D\left[-5(h M+2 \sigma)^{2}+2 h^{3} D\right]=0 .
\end{aligned}
$$

This is the blue line appearing in Figure 14.

(ii) When a valid root of the cubic polynomial is 0 , then a relative equilibrium in the plateau collides with the peak at $a_{1}=0$. So a bifurcation involving $P_{2}$ happens when

$$
\Gamma_{2} \equiv(h M+2 \sigma)^{2}-2 h^{3} D=0 .
$$



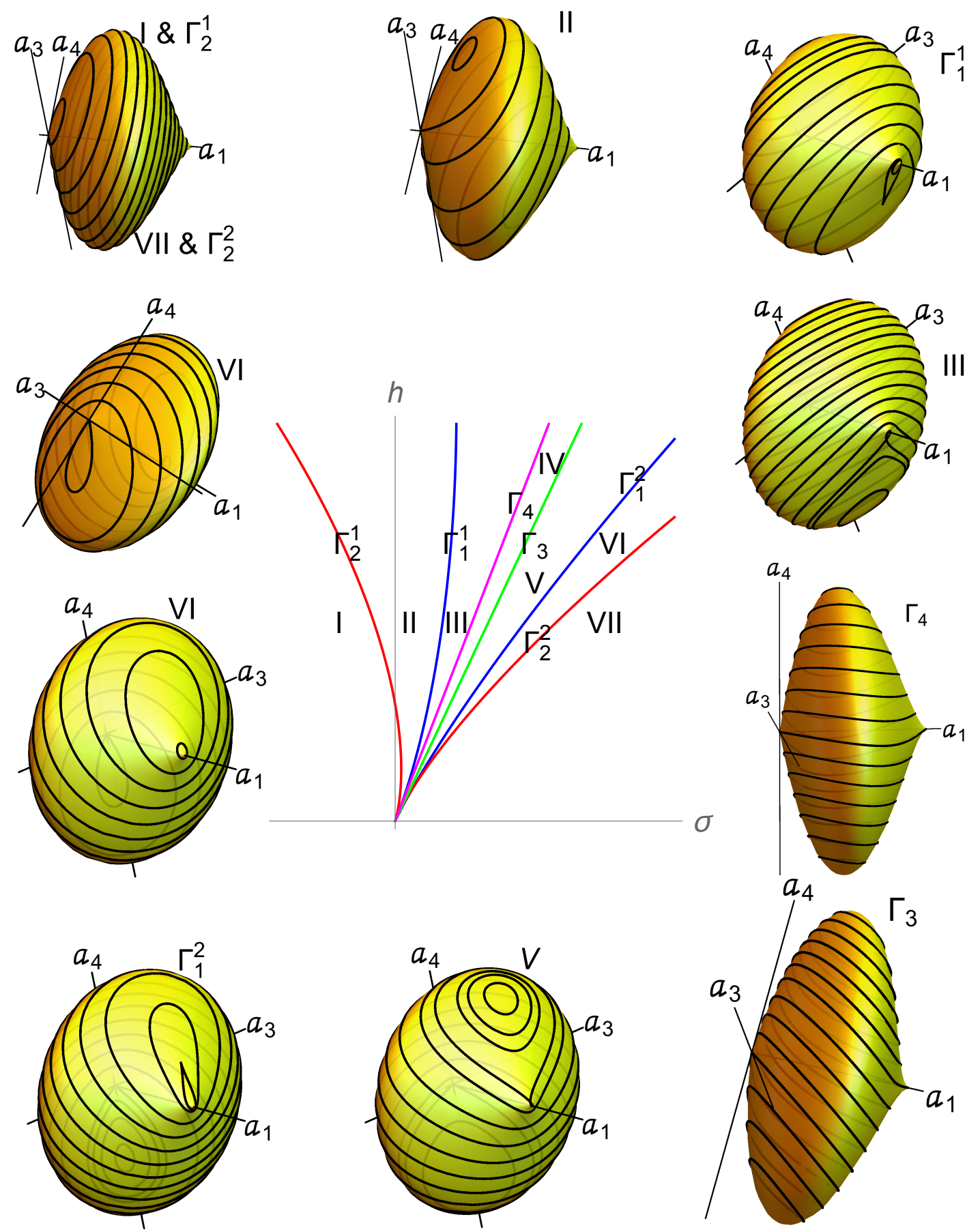

FIGURE 14. Bifurcation plane and flows for the detuning of the $3: 2$ resonance. In each picture of the orbit space one can also see the flow in the back

This is the red line appearing in Figure 14.

(iii) When a valid root of the cubic polynomial is $h / 3$, then a relative equilibrium in the plateau collides with the peak at $a_{1}=h / 3$. Thus a bifurcation involving $P_{1}$ takes place when

$$
\Gamma_{3} \equiv \Delta h+3(h M+2 \sigma)=0 .
$$


This is the green line appearing in Figure 14.

7.3.2. Bifurcations and Flow of the Full System. We start by applying the symplectic smoothing technique in order to unfold the peak points. For $P_{2}$ we use formulae (29) while for $P_{1}$ we apply (31). Specifically for the peak at $a_{1}=0$ we get

$$
\begin{array}{rlrl}
a_{1} & =x^{2}+y^{2}, & a_{2} & =\frac{1}{2}\left[h-3\left(x^{2}+y^{2}\right)\right], \\
a_{3}=\frac{1}{2 \sqrt{2}}\left(x^{2}-y^{2}\right)\left[h-3\left(x^{2}+y^{2}\right)\right]^{3 / 2}, & a_{4} & =-\frac{1}{\sqrt{2}} x y\left[h-3\left(x^{2}+y^{2}\right)\right]^{3 / 2}
\end{array}
$$

while for the peak at $a_{1}=h / 3$, the change is

$$
\begin{array}{llrl}
a_{1} & =\frac{1}{3}\left[h-2\left(x^{2}+y^{2}\right)\right], & a_{2} & =x^{2}+y^{2}, \\
a_{3}=\frac{1}{3} x\left(x^{2}-3 y^{2}\right)\left[h-2\left(x^{2}+y^{2}\right)\right], & a_{4}=\frac{1}{3} y\left(3 x^{2}-y^{2}\right)\left[h-2\left(x^{2}+y^{2}\right)\right] .
\end{array}
$$

Now we plug (49) and (50) in $\bar{H} / 2$ and obtain the reduced Hamiltonian around the peaks. Linearizing the Hamiltonian around $(0,0)$ for the two peaks we compute their eigenvalues, arriving at

$$
\nu_{2}^{ \pm}= \pm \frac{1}{4} \sqrt{2 h^{3} D-(h M+2 \sigma)^{2}}, \quad \nu_{1}^{ \pm}= \pm \frac{1}{18}[-\Delta h-3(h M+2 \sigma)] \mathrm{i},
$$

the first one applies for $P_{2}$ and the second one for $P_{1}$. It is immediate to deduce that $P_{2}$ is hyperbolic when $2 h^{3} D-(h M+2 \sigma)^{2}>0$ and elliptic when $2 h^{3} D-(h M+2 \sigma)^{2}<0$. This expression vanishes on the curve $\Gamma_{2}$. Regarding $P_{1}$, it is a center (elliptic) provided $\Delta h+3(h M+2 \sigma)$ does not vanish, but this is precisely the expression of $\Gamma_{3}$.

The peak points are reconstructed as periodic solutions of the full system in $\mathbb{R}^{4}$ of periods near $\pi$ for $P_{2}$ and near $2 \pi / 3$ for $P_{1}$. If we apply Theorem 6.4 , the approximate non-trivial characteristic multipliers of the periodic solution related to $P_{2}$ are $e^{(3 / 2) 2 \pi \mathrm{i}}\left[1+(2 \pi / 2) \varepsilon^{2} \nu_{2}^{+}\right]$and $e^{-(3 / 2) 2 \pi \mathrm{i}}\left[1+(2 \pi / 2) \varepsilon^{2} \nu_{2}^{-}\right]$thence $-1 \pm(\pi / 4) \varepsilon^{2} \sqrt{2 h^{3} D-(h M+2 \sigma)^{2}}$. The multipliers of the periodic solution related to $P_{1}$ are $e^{(2 / 3) 2 \pi \mathrm{i}}[1+$ $\left.(2 \pi / 3) \varepsilon^{2} \nu_{1}^{+}\right]=-(1+\sqrt{3} \mathrm{i}) / 2-[(\sqrt{3}-\mathrm{i}) / 54] \pi \varepsilon^{2}[\Delta h+3(h M+2 \sigma)]$ and $e^{-(2 / 3) 2 \pi \mathrm{i}}\left[1+(2 \pi / 3) \varepsilon^{2} \nu_{1}^{-}\right]=$ $-(1-\sqrt{3} \mathrm{i}) / 2-[(\sqrt{3}+\mathrm{i}) / 54] \pi \varepsilon^{2}[\Delta h+3(h M+2 \sigma)]$, thus the stability of these periodic solutions depends basically on the eigenvalues $\nu_{1}^{ \pm}, \nu_{2}^{ \pm}$, hence on the parameters involved in the study. We shall be more specific in the next paragraphs.

Now we can deal with the bifurcation analysis. To simplify the presentation a bit we fix specific values of the parameters $\Delta, M$ and $D$ and draw a bifurcation plane for $\sigma$ and $h$, which are the significant parameters, see Figure 14. The main feature when varying the values of $\Delta, M$ and $D$ is that the sequence of bifurcations is the same but occurs in a different order. In Figure 14 we also present a sketch of the flow of the reduced system throughout all regions and bifurcation lines. In the following paragraphs we describe the evolution of the flow starting from the left in the bifurcation plane and going on to the right. In the flow pictures we start by the top left and continue clockwise.

In region $I$ the only relative equilibria are the peaks $P_{1}$ and $P_{2}$ and both are elliptic points.

On the first branch of the red line that we call $\Gamma_{2}^{1}$ a bifurcation involving $P_{2}$ happens though on the line $P_{2}$ and $P_{1}$ are still centers. After crossing this line, when being in region II, $P_{2}$ changes from elliptic to hyperbolic and the stable equilibrium $L_{1}$ in the plateau will emerge from it. The peak $P_{1}$ stays stable. The bifurcation occurring on $\Gamma_{2}^{1}$ is a supercritical Hamiltonian flip bifurcation and can be analyzed analogously as we did in Section 7.2. In particular $L_{1}$ reconstructs to an elliptic periodic solution of period near $2 \pi$ and a Hamiltonian flip bifurcation of periodic solutions occurs for values of the parameters with $\Gamma_{2}^{1} \approx 0$.

On the first branch of the blue curve, i.e. $\Gamma_{1}^{1}$, a center-saddle bifurcation in the plateau takes place. A cusp $L_{2}$ appears near the peak $P_{1}$, that continues to be stable. The points $P_{2}$ and $L_{1}$ remain hyperbolic and elliptic respectively. The coordinate $a_{1}^{0}$ of $L_{2}$ is obtained as a solution of $p_{3}\left(a_{1}\right)=0$.

After crossing the bifurcation curve, in region III, the cusp $L_{2}$ in the plateau becomes a saddle and a new stable equilibrium $L_{3}$ appears from it in the plateau. The center-saddle bifurcation in $\mathbb{O}$ involving $L_{2}$, $L_{3}$ is reconstructed as a center-saddle bifurcation of periodic solutions for the Hamiltonian $H$ with $\Gamma_{1}^{1} \approx 0$, 
in a similar way to the analysis of the 2:-1 resonance of Section 7.2. Thus $L_{2}, L_{3}$ correspond to periodic solutions of $H$ with periods near $2 \pi$. The stability of $P_{1}, P_{2}$ and $L_{1}$ is the same as in region II and on $\Gamma_{1}^{1}$.

On the purple line, $\Gamma_{4}$, the energy of the saddle $L_{2}$ is the same as the energy of $P_{2}$ and there is a connection of saddles, that is a global bifurcation. The line $\Gamma_{4}$ has been obtained numerically. We present in Figure 15 a view of the reduced flow near $P_{2}$ in the coordinates $x, y$ of (49). Due to the $2: 1$ covering $(p=2)$ all the equilibria in the picture excepting the origin appear duplicated, thus the two saddles placed outside the point $(0,0)$ correspond to the point $L_{2}$. Since the eigenvalues of the corresponding Hessian at the points $P_{2}$ and $L_{2}$ do not vanish at $\Gamma_{4}$ a global bifurcation of hyperbolic periodic solutions occurs for the full system $H$ in $\mathbb{R}^{4}$.

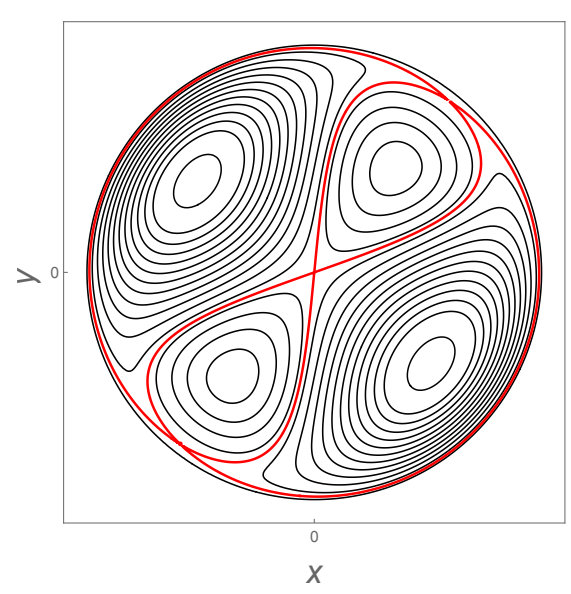

FIGURE 15. Unfolding of the 3:2 resonance: projection of a neighborhood of the point $\left(a_{1}, a_{3}, a_{4}\right)=$ $(0,0,0)$ in the $(x, y)$-plane. The red curve corresponds to $\Gamma_{4}$, i.e. to the connection between the two saddle points in $a$-space

In region $I V$ the number of equilibria together with their stability is the same as in region III but, out of the global bifurcation, the stable equilibrium $L_{1}$ (that was attached to $P_{2}$ ) is now attached to the saddle $L_{2}$, and the stable equilibrium $L_{3}$, that was attached to $L_{2}$, is now attached to $P_{2}$.

On $\Gamma_{3}$ a bifurcation of $P_{1}$ occurs. The saddle $L_{2}$ in the plateau collides with $P_{1}$ (which was an elliptic point) and disappears while $P_{1}$ becomes a cusp. The stable equilibrium $L_{1}$ is attached to $P_{1}$ and the peak $P_{2}$ continues to be hyperbolic and the stable equilibrium $L_{3}$ is linked to it. From this point on, we continue by reversing all the bifurcations. In region $V$ the peak $P_{1}$ turns into a center and a new saddle $L_{4}$ appears. The bifurcation involving $P_{1}, L_{2}$ and $L_{4}$ is studied with the Hamiltonian $\bar{H}$ in the coordinates $x, y$ defined through (50). Thus the peak $P_{1}$ behaves like a 3-bifurcation point, see $[39,43]$ due to the $3: 1$ covering $(q=3)$ caused by the transformation (50). The rest of points keep on their stability character. The point $L_{4}$ gives rise to an unstable periodic solution in $\mathbb{R}^{4}$ of period near $2 \pi$. This bifurcation translates into a bifurcation of periodic solutions of the same type that takes place for the Hamiltonian (47) when $\Gamma_{3} \approx 0$.

On the second branch of the blue line, $\Gamma_{1}^{2}$, a center-saddle bifurcation in the plateau occurs. The center $L_{1}$ collides with the saddle $L_{4}$ and becomes a cusp point. Once in region $V I$ the cusp in the plateau vanishes while $P_{1}$ remains elliptic, $P_{2}$ hyperbolic and $L_{3}$ elliptic. Then a center-saddle bifurcation of periodic solutions occurs for $H$ given in (47) when $\Gamma_{1}^{2} \approx 0$.

In the second branch of the red line, $\Gamma_{2}^{2}$, a bifurcation of $P_{2}$ occurs. The elliptic point in the plateau, $L_{3}$, collides with $P_{2}$ and the peak becomes stable. The other peak, $P_{1}$, remains stable. This is a Hamiltonian flip bifurcation involving $P_{2}$ and $L_{3}$ that translates into a Hamiltonian flip bifurcation of periodic solutions for the full system.

Finally in region VII the situation is the same as in region I, the peaks are the only critical points and they are elliptic. 


\section{ACKNowledgments}

The authors are partially supported by Projects MTM 2011-28227-C02-01 of the Ministry of Science and Innovation of Spain, MTM 2014-59433-C2-1-P of the Ministry of Economy and Competitiveness of Spain and by the Charles Phelps Taft Foundation.

\section{REFERENCES}

[1] R. Abraham and J. E. Marsden, Foundations of Mechanics, Benjamin/Cummings Publishing Co., Inc., Advanced Book Program, Reading, Ma., 1978.

[2] J. P. Amiet and S Weigert, Commensurate harmonic oscillators: Classical symmetries, J. Math. Phys. 43 (8), (2002), 4110-4126.

[3] J. M. Arms, R. H. Cushman and M. J. Gotay, A universal reduction procedure for Hamiltonian group actions, in: The Geometry of Hamiltonian Systems, T. Ratiu, ed., Springer-Verlag, New York, 1991, pp. 33-51.

[4] M. Beltrametti and L. Robbiano, Introduction to the theory of weighted projective spaces, Expo. Math. 4 (2) (1986), 111-162.

[5] T. M. Cherry, Some examples of trajectories defined by differential equations of a generalised dynamical type, Trans. Camb. Phil. Soc. XXIII, 1925, 169-200.

[6] R. C. Churchill, M. Kummer and D. L. Rod, On averaging, reduction, and symmetry in Hamiltonian systems, J. Differential Equations 49 (3), 1983, 359-414.

[7] D. Cox, J. Little and D. O'Shea, Ideals, Varieties, and Algorithms. An Introduction to Computational Algebraic Geometry and Commutative Algebra, $3^{\text {nd }}$ Ed., Springer-Verlag, New York, Berlin, Heidelberg, 2007.

[8] R. H. Cushman and L. M. Bates, Global Aspects of Classical Integrable Systems, Birkhäuser Verlag, Basel, 1997.

[9] R. H. Cushman, H. R. Dullin, H. Hanßmann and S. Schmidt, The 1: \pm 2 resonance, Regul. Chaotic Dyn. 12 (6), 2007, 642-663.

[10] A. Deprit, Canonical transformations depending on a small parameter, Celestial Mech. 1 (1), 1969, 12-30.

[11] H. Derksen and G. Kemper, Computational Invariant Theory, Encyclopaedia of Mathematical Sciences, Invariant Theory and Algebraic Transformation Groups I, Springer-Verlag, New York, 2002.

[12] J. J. Duistermaat, Bifurcations of periodic solutions near equilibrium points of Hamiltonian system, in: Bifurcation Theory and Applications, Montecatini 1983, L. Salvadori, ed., Lecture Notes in Mathematics 1057, Springer-Verlag, Berlin, Heidelberg, 1984, pp. 55-105.

[13] H. R. Dullin, H. E. Lomelí and J. D. Meiss, Symmetry reduction by lifting for maps, Nonlinearity 25 (6), 2012 , 1709-1733.

[14] A. S. Egilsson, Linear Hamiltonian circle actions that generate minimal Hilbert bases, Ann. Inst. Fourier (Grenoble) $\mathbf{5 0}(1), 2000,285-315$.

[15] M. El Samaloty, Averaging and Bifurcation Theory, Ph.D. Thesis, University of Cincinnati, 1984.

[16] A. Elipe, Complete reduction of oscillators in resonance p:q, Phys. Rev. E 61 (6), 2000, 6477-6484.

[17] _ Extended Lissajous variables for oscillators in resonance, Math. Comput. Simulation 57 (3-5), 2001, $217-226$.

[18] A. Elipe, V. Lanchares and A. I. Pascual, On the stability of equilibria in two-degrees-of-freedom Hamiltonian systems under resonances, J. Nonlinear Sci. 15 (5), 2005, 305-319.

[19] N. W. Evans, Superintegrability in classical mechanics, Phys. Rev. A 41 (10), 1990, 5666-5676.

[20] F. Fassò, Superintegrable Hamiltonian systems: geometry and perturbations, Acta Appl. Math. 87 (1-3), 2005, 93-121.

[21] S. Ferrer, J. F. Palacián and P. Yanguas, Hamiltonian oscillators in 1:1:1 resonance: normalization and integrability, J. Nonlinear Sci. 10 (2), 2000, 145-174.

[22] A. T. Fomenko, Topological Classification of Integrable Systems, Advances in Soviet Mathematics 6, American Mathematical Society, Providence, RI, 1991.

[23] G. Haller, Chaos Near Resonance, Appl. Math. Sci. 138, Springer-Verlag, New York, 1999.

[24] G. Haller and S. Wiggins, Geometry and chaos near resonant equilibria of 3-DOF Hamiltonian systems, Phys. D 90 (4), 1996, 319-365.

[25] H. Hanßmann, Local and Semi-Local Bifurcations in Hamiltonian Dynamical Systems: Results and Examples, Lecture Notes in Mathematics 1893, Springer-Verlag, Berlin, Heidelberg, 2007.

[26] _ Perturbations of superintegrable systems, Acta Appl. Math. 137 (1), 2015, 79-95.

[27] J. Henrard, Lyapunov's center theorem for resonant equilibrium, J. Differential Equations 14 (3), 1973, 431-441.

[28] D. Holm, Geometric Mechanics, Part 1: Dynamics and Symmetry, Imperial College Press, London, 2008.

[29] D. Holm and C. Vizman, Dual pairs in resonances, J. Geom. Mech. 4 (3), 2013, 297-311.

[30] I. Hoveijn and F. Verhulst, Chaos in the 1:2:3 Hamiltonian normal form, Phys. D 44 (3), 1990, 397-406.

[31] M. Kummer, An interaction of three resonant modes in a nonlinear lattice, J. Math. Anal. Appl. 52 (1), 1975, 64-104.

[32] $\quad$ On the construction of the reduced phase space of a Hamiltonian system with symmetry, Indiana Univ. Math. J. 30 (2), 1981, 281-291. 
[33] _ On resonant Hamiltonian systems with finitely many degrees of freedom, in: Local and Global Methods of Nonlinear Dynamics, A. W. Sáenz, W. W. Zachary and R. Cawley, eds., Lecture Notes in Physics 252, SpringerVerlag, Berlin Heidelberg, 1986, pp. 19-31.

[34] _ On resonant classical Hamiltonians with $n$ frequencies, J. Differential Equations 83 (2), 1990, 220-243.

[35] E. Lerman, R. Montgomery and R. Sjamaar, Examples of singular reduction, in: Symplectic Geometry (Warwick, 1990), D. Salamon, ed., London Mathematical Society Lecture Note Series, vol. 192, Cambridge University Press, Cambridge, 1993, pp. 127-155.

[36] E. Lerman and S. Tolman, Hamiltonian torus actions on symplectic orbifolds and toric varieties, Trans. Amer. Math. Soc. 349 (10), 1997, 4201-4230.

[37] L. Markus and K. R. Meyer, Generic Hamiltonian dynamical systems are neither integrable nor ergodic, Mem. Amer. Math. Soc. 144, 1974.

[38] J. Marsden and A. Weinstein, Reduction of symplectic manifolds with symmetry, Rep.Math. Phys. 5 (1), 1974 , $121-130$.

[39] K. R. Meyer, Generic bifurcation of periodic points, Trans. Amer. Math. Soc. 149, 1970, 95-107.

[40] - Symmetries and integrals in mechanics, in: Dynamical Systems, M. M. Peixoto, ed., Acad. Press, New York, 1973, pp. 259-272.

[41] _ Lie transform tutorial - II, in: Computer Aided Proofs in Analysis, K. R. Meyer and D. S. Schmidt, eds., The IMA Volumes in Mathematics and its Applications 28, Springer-Verlag, Berlin and New York, 1991, pp. 190-210.

[42] _ Periodic Solutions of the N-Body Problem, Lecture Notes in Mathematics 1719, Springer-Verlag, Berlin, Heidelberg, 1999.

[43] K. R. Meyer and D. Offin, Introduction to Hamiltonian Dynamical Systems and the N-Body Problem, $3^{\text {rd }}$ Ed., Appl. Math. Sci. 90, Springer, New York, 2017.

[44] K. R. Meyer, J. F. Palacián and P. Yanguas, Geometric averaging of Hamiltonian systems: periodic solutions, stability, and KAM tori, SIAM J. Appl. Dyn. Syst. 10 (3), 2011, 817-856.

[45] — The elusive Liapunov periodic solutions, Qual. Theory Dyn. Syst. 14 (2), 2015, 381-401.

[46] Singular reduction of high dimensional Hamiltonian systems, in preparation.

[47] J. Moser, Regularization of Kepler's problem and the averaging method on a manifold, Comm. Pure Appl. Math. 23 (4), 1970, 609-636.

[48] J. Palacián and P. Yanguas, Reduction of polynomial planar Hamiltonians with quadratic unperturbed part, SIAM Rev. 42 (4), 2000, 671-691.

[49] T. Poston and I. Stewart, Catastrophe Theory and Its Applications, Pitman, Boston, 1978.

[50] G. Reeb, Sur certaines propriétés topologiques des trajectoires des systèmes dynamiques, Acad. Roy. Belg. Cl. Sci. Mém. Collect. $8^{\circ}$ (2) $\mathbf{2 7}$ (9), 1952.

[51] J. A. Sanders, Are higher order resonances really interesting?, Celestial Mech. 16 (4), 1978, 421-440.

[52] _ Normal forms of 3 degree of freedom Hamiltonian systems at equilibrium in the resonant case, in: Global Integrability of Field Theories, J. Calmet, W. M. Seiler and R. W. Tucker, eds., Univ. Karlsruhe, Karlsruhe, 2006, pp. 335-346.

[53] J. A. Sanders, F. Verhulst and J. Murdock, Averaging Methods in Nonlinear Dynamical Systems, $2^{\text {nd }}$ Ed., Appl. Math. Sci. 59, Springer, New York, 2007.

[54] I. Satake, On a generalization of the notion of a manifold, Proc. Natl. Acad. Sci. USA 42, 1956, 359-363.

[55] D. S. Schmidt, Periodic solutions near a resonant equilibrium of a Hamiltonian system, Celestial Mech. 9 (1), 1974, 81-103.

[56] S. Schmidt and H. R. Dullin, Dynamics near the p:-q resonance, Phys. D 239 (19), 2010, 1884-1891.

[57] R. Sjamaar and E. Lerman, Stratified symplectic spaces and reduction, Ann. Math. 134 (2), 1991, 375-422.

[58] E. van der Aa, First-order resonances in three-degrees-of-freedom systems, Celestial Mech. 31 (2), 1983, $163-191$.

[59] E. van der Aa and M. de Winkel, Hamiltonian systems in 1:2: $\omega$-resonance ( $\omega=5$ or 6), Int. J. Non-Linear Mech. 29 (2), 1994, 261-270.

[60] E. T. Whittaker, A Treatise on the Analytical Dynamics of Particles and Rigid Bodies, Cambridge University Press, Cambridge, 1904.

[61] P. Yanguas, J. F. Palacián, K. R. Meyer and H. S. Dumas, Periodic solutions in Hamiltonian systems, averaging, and the lunar problem, SIAM J. Appl. Dyn. Syst. 7 (2), 2008, 311-340.

Department of Mathematical Sciences, University of Cincinnati, Cincinnati, Ohio 45221-0025

E-mail address: ken.meyer@uc.edu

Departamento de Ingeniería Matemática e Informática and Institute for Advanced Materials (INAMAT), Universidad Pública de Navarra, 31006 Pamplona, Spain

E-mail address: palacian@unavarra.es

Departamento de Ingeniería Matemática e Informática and Institute for Advanced Materials (INAMAT), Universidad Pública de Navarra, 31006 Pamplona, Spain

E-mail address: yanguas@unavarra.es 